\title{
Flavour Expedition to the Zeptouniverse
}

\author{
Andrzej J. Buras ${ }^{* \dagger}$ \\ TUM-IAS, Lichtenbergstr. 2a, D-85748 Garching, Germany \\ Technical University Munich, Physics Department, D-85748 Garching, Germany, \\ E-mail: aburaseph.tum.de
}

\begin{abstract}
After the completion of the Standard Model (SM) through the Higgs discovery in 2012 particle physicists are waiting for the discovery of new particles either directly with the help of the Large Hadron Collider (LHC) or indirectly through quantum fluctuations causing certain rare processes to occur at different rates than predicted by the SM. While the later route is very challenging, requiring very precise theory and experiment, it allows in principle a much higher resolution of short distance scales than it is possible with the help of the LHC. In fact in the coming flavour precision era, in which the accuracy of the measurements of rare processes and of the relevant lattice QCD calculations will be significantly increased, there is a good chance that we may get an insight into the scales as short as $10^{-21} \mathrm{~m}$ (Zeptouniverse) corresponding to energy scale of $200 \mathrm{TeV}$ or even shorter distance scales. We discuss the requirements that have to be met for such a flavour expedition to the Zeptouniverse to be successful. In particular we emphasize the power of correlations between flavour observables in the search for New Physics (NP) and identify a number of correlations that could allow to discover NP even if it would appear at the level of $20 \%$ of the SM contributions. The correlation between $\mathscr{B}\left(K^{+} \rightarrow \pi^{+} v \bar{v}\right), \overline{\mathscr{B}}\left(B_{s} \rightarrow\right.$ $\mu^{+} \mu^{-}$) and $\gamma$ extracted from tree-level decays within the SM is one of them. After presenting the simplest correlations in CMFV and $U(2)^{3}$ models we address the recent data on $B_{s, d} \rightarrow \mu^{+} \mu^{-}$ and the anomalies in $B_{d} \rightarrow K\left(K^{*}\right) \mu^{+} \mu^{-}$, including breakdown of lepton flavour universality, in the context of $Z^{\prime}$-models with quark flavour violating neutral couplings. A brief discussion of leptoquark models is also given. We emphasize the correlations of $B_{d} \rightarrow K\left(K^{*}\right) \mu^{+} \mu^{-}$with $B_{d} \rightarrow$ $K\left(K^{*}\right) v \bar{v}$ that allow to distinguish between various explanations of the anomalies in question. Finally we summarize the recent study of $K \rightarrow \pi \nu \bar{v}$ and $B_{d, s} \rightarrow \mu^{+} \mu^{-}$decays which demonstrates that these decays play important roles in finding out what happens in the Zeptouniverse.
\end{abstract}

Flavorful Ways to New Physics

28-31 October 2014

Freudenstadt - Lauterbad, Germany

\footnotetext{
*Speaker.

${ }^{\dagger}$ FLAVOUR(267104)-ERC-90
} 


\section{Overture}

In spite of tremendous efforts of experimentalists and theorists to find New Physics (NP) beyond the Standard Model (SM), no clear indications for NP beyond dark matter, neutrino masses and matter-antimatter asymmetry in the universe have been observed. Yet, the recent discovery of a Higgs-like particle and the overall agreement of the SM with the present data shows that our general approach of describing physics at very short distance scales with the help of exact (QED and QCD) and spontaneously broken (for weak interactions) gauge theories is correct.

As the SM on the theoretical side is not fully satisfactory and the three NP signals mentioned above are already present, we know that some new particles and new forces have to exist, hopefully within energy scales being presently directly explored by the LHC or not far above them. The upgrade in the energy of the LHC, the upgrade of the LHCb, SuperKEKB and dedicated kaon physics experiments at CERN and J-PARC, as well as improved measurements of charged lepton flavour violation (CLFV), electric dipole moments (EDMs) and $(g-2)_{\mu, e}$ will definitely shed light on the question whether NP is present below, say, $10 \mathrm{TeV}$. However in the coming decades only rare processes will allow us to go beyond $10 \mathrm{TeV}$. These are in particular particle-antiparticle mixings ( $\Delta F=2$ processes), rare decays of mesons ( $\Delta F=1$ processes), CLFV, EDMs and $(g-2)_{\mu, e}$. As this is an indirect search for NP one has to develop special strategies to reach the Zeptouniverse, that is scales as short as $10^{-21} \mathrm{~m}$ or equivalently energy scales as high as several hundreds of TeV. The present lecture discusses some of such strategies developed in my group at the Technical University in Munich during last ten years. They are summarized in [1] and in my talk at EPSHEP13 in Stokholm [2] which I opened with a similar overture as the general situation as far as NP is concerned has not changed by much since then. We are still waiting for NP.

Yet, the rest will include new results and the goals will be presented in a bit different manner even if some overlap with [2] is unavoidable. In any case it is unquestionable that NP beyond the SM exists and it is our duty to search for it, not only through high energy collisions at the LHC, but in particular through rare transitions both in the quark and lepton sectors that we listed above. This is not only because we would like to know which new creatures exist down there but in particular in order to answer many open questions which I will not repeat here as they are known to every particle physicist. But let me still reemphasize that the issue of the origin of flavour plays a very important role in these efforts for a very obvious reason. In our search for a more fundamental theory we need to improve our understanding of flavour.

While we mentioned above that the SM describes the present data well, there are several signals in the present flavour data which give us hopes that the increase of precision in experiment and theory in the coming years could indeed provide valuable information about the nature of NP beyond the SM. It is strategically useful to list already here these departures from SM expectations, write a few lines about the possible NP behind them and elaborate on some of them later. These are:

1. Anomalies in angular observables in $B_{d} \rightarrow K^{*} \mu^{+} \mu^{-}$and in the branching ratio $\mathscr{B}\left(B_{d} \rightarrow\right.$ $K^{*} \mu^{+} \mu^{-}$) that is found to be smaller than predicted within the SM. A heavy neutral gauge boson $Z^{\prime}$ or leptoquarks with particular tranformation properties under the SM gauge group are the leading candidates among the ones proposed as the origin of these deviations.

2. Breakdown of lepton flavour universality in $B^{+} \rightarrow K^{+} \ell^{+} \ell^{-}$with the branching ratio for 
$B^{+} \rightarrow K^{+} e^{+} e^{-}$in agreement with the SM but $\mathscr{B}\left(B^{+} \rightarrow K^{+} \mu^{+} \mu^{-}\right)$found to be smaller than predicted by the SM. Again a heavy $Z^{\prime}$ or leptoquarks are the simplest explanations of this behaviour.

3. The ratio

$$
\frac{\mathscr{B}\left(B_{d} \rightarrow \mu^{+} \mu^{-}\right)}{\mathscr{B}\left(B_{s} \rightarrow \mu^{+} \mu^{-}\right)}=(4.8 \pm 2.2)\left[\frac{\mathscr{B}\left(B_{d} \rightarrow \mu^{+} \mu^{-}\right)}{\mathscr{B}\left(B_{s} \rightarrow \mu^{+} \mu^{-}\right)}\right]_{\mathrm{SM}}
$$

is found to be larger than predicted in the SM. Interestingly, as we will see below, while $\mathscr{B}\left(B_{s} \rightarrow\right.$ $\left.\mu^{+} \mu^{-}\right)$appears to be smaller than its SM value, $\mathscr{B}\left(B_{d} \rightarrow \mu^{+} \mu^{-}\right)$is measured to be larger. This pattern violates the predictions not only of the SM but of the full class of models with minimal flavour violation (MFV) where the enhancement (suppression) of one of these branching ratios implies uniquely enhancement (suppression) of the other one. Here again a $Z^{\prime}$ can help [1].

4. Moderate tensions between $K^{0}-\bar{K}^{0}$ and $B_{s, d}^{0}-\bar{B}_{s, d}^{0}$ mixing observables which have been with us already since $2008[3,4]$. Typically either the CP asymmetry $S_{\psi K_{S}}$ is above the data or $\varepsilon_{K}$ is below the data. Moreover, if one increases $\left|V_{c b}\right|$ to get a better result for $\varepsilon_{K}$, the mass differences $\Delta M_{s, d}$ are shifted above the data. But the uncertainties in the relevant $\hat{B}_{i}$ parameters are too large to claim the presence of NP. Future lattice QCD calculations and in particular improved determination of the CKM parameters will clarify this ${ }^{1}$. This is important as these tensions signal the presence of NP beyond MFV. While $Z^{\prime}$ could also help here, heavy neutral scalars or the reduction of flavour symmetry of MFV models $\left(U(3)^{3}\right)$ down to $U(2)^{3}$ would also work. However, $U(2)^{3}$ models cannot help with the anomalies 1-3 listed above and in the case of $\mathbf{1}$ and $\mathbf{2}$ this also applies to heavy neutral scalars.

5. Significant departures from SM expectations in $B_{d} \rightarrow D \tau \nu_{\tau}$ and $B_{d} \rightarrow D^{*} \tau \nu_{\tau}$ signaling possibly the presence of new heavy charged scalars or gauge bosons.

6. The $(g-2)_{\mu}$ anomaly which was with us for more than ten years.

In this lecture I will have nothing to add about the last two tensions to what is known already in the literature and I refer to the review [1] where brief summaries of these two topics together with the relevant literature can be found. Similar, I have presently nothing to add to various possible departures from SM expectations in non-leptonic decays of $B$ and $D$ mesons, partly because theoretical uncertainties in these decays are larger than in the processes discussed below. Yet, it should be emphasized that in the flavour precision era, in which the measurements of a multitude of non-leptonic decays will be very much improved, also these decays could be very useful, in particular in the tests of CP violation [6].

\section{Basic Requirements for Reaching the Zeptouniverse}

The coming ten years (2015-2025) of flavour precision era invites us to attempt an expedition from the Attouniverse to the Zeptouniverse. For such an expedition to have a chance to be successful at least the following requirements have to be fullfiled:

- Many precise measurements of many observables.

\footnotetext{
${ }^{1}$ As the $\hat{B}_{K}$ parameter, relevant for $\varepsilon_{K}$, is already precisely known and the lattice analyses favour significantly lower values of $\left|V_{c b}\right|$ and $\left|V_{u b}\right|$ than inclusive determinations, from the point of view of lattice QCD, SM value of $\varepsilon_{K}$ turns out to be by $3 \sigma$ below the data [5].
} 
- Precise extraction of CKM parameters from tree level decays which are expected to have at most tiny NP contributions ${ }^{2}$. Here the main targets for coming years are

$$
\left|V_{u b}\right|, \quad\left|V_{c b}\right|, \quad \gamma
$$

where $\gamma$, one of the angles in the Unitarity Triangle, is up to the sign the complex phase of $V_{u b}$.

- Precise lattice QCD calculations of weak decay constants $F_{B_{s}}$ and $F_{B_{d}}$, of various nonperturbative parameters $\hat{B}_{i}$ and of form factors for various semi-leptonic transitions, in particular for $B \rightarrow K\left(K^{*}\right)$ transitions with leptons in the final state and those relevant for the determinations of $\left|V_{u b}\right|$ and $\left|V_{c b}\right|$. For a review see [10] and references therein. In the case of non-leptonic decays of mesons lattice QCD appears to be less useful and in this case approaches like QCD factorization approach [11] and those based on flavour symmetries and their breakdown [6] will continue to play important roles.

- NLO and NNLO QCD corrections and NLO electroweak corrections to various Wilson coefficients. Among the tasks listed here I would claim that at least within the SM this task has been completed after 26 years of efforts by several theorists (1988-2014). An updated review of these efforts can be found in [12]. I do not think we need more precision here within the SM and these calculations are sufficiently demanding that there is no point in doing them in extensions of the SM before we know what nature is telling us about NP. An exception are tree-level flavour changing neutral currents mediated by $Z^{\prime}, Z$ or heavy neutral scalars. Their structure is sufficiently simple so that NLO QCD corrections to these exchanges could be easily calculated $[13,14]$.

Concerning the second item one needs the clarification of the discrepancies between inclusive and exclusive determinations of $\left|V_{c b}\right|$ and $\left|V_{u b}\right|$ from tree-level decays $[15,16]$. The present status of these determinations is as follows. From inclusive determinations based dominantly on heavy quark expansions one has $[17,18]$

$$
\left|V_{u b}\right|_{\text {incl }}=(4.40 \pm 0.25) \cdot 10^{-3}, \quad\left|V_{c b}\right|_{\text {incl }}=(42.21 \pm 0.78) \cdot 10^{-3}
$$

whereas from exclusive determinations based dominantly on formfactors from lattice QCD one has [18-20]

$$
\left|V_{u b}\right|_{\mathrm{excl}}=(3.72 \pm 0.14) \cdot 10^{-3}, \quad\left|V_{c b}\right|_{\mathrm{excl}}=(39.36 \pm 0.75) \cdot 10^{-3}
$$

These differences introduce significant uncertainties in SM predictions for rare $K$ and $B_{s, d}$ decays, which have to be decreased by much if we want to study NP efficiently. In particular rare decays $K^{+} \rightarrow \pi^{+} v \bar{v}$ and $K_{L} \rightarrow \pi^{0} v \bar{v}$ and $\varepsilon_{K}$ are very sensitive to the value of $\left|V_{c b}\right|$ but also the branching ratios for $B_{s, d} \rightarrow \mu^{+} \mu^{-}$and the mass differences $\Delta M_{s, d}$ depend quadratically on it. It is likely that this problem will be resolved only by Belle II experiment at SuperKEKB at the end of this decade but one should hope that further theoretical efforts will tell us what is really going on. From the

\footnotetext{
${ }^{2}$ Recent analyses of the room left for NP in tree-level decays can be found in [7-9].
} 
analyses in [21,22] it is unlikely that NP, like right-handed charged currents, are responsible for these discrepancies but this should be further clarified.

For the time being one can take a weighted average of these results and scale the errors based on the resulting $\chi^{2}$, which gives [23]

$$
\left|V_{u b}\right|_{\text {avg }}=(3.88 \pm 0.29) \times 10^{-3}, \quad\left|V_{c b}\right|_{\text {avg }}=(40.7 \pm 1.4) \times 10^{-3} .
$$

For the CKM angle $\gamma$ the current world average of direct measurements [24] reads

$$
\gamma=\left(73.2_{-7.0}^{+6.3}\right)^{\circ}
$$

The fourth element of the CKM matrix is already very well known

$$
\left|V_{u s}\right|=0.2252 \pm 0.0009 \text {. }
$$

Concerning QCD lattice calculations of non-perturbative parameters relevant for $\Delta F=2$ transitions and of formfactors entering rare decays like $B_{d} \rightarrow K\left(K^{*}\right) \ell^{+} \ell^{-}$and $B_{d} \rightarrow K\left(K^{*}\right) v \bar{v}$, where in addition to lattice QCD also light-cone sum rules (LCSR) [25] play an important role, I am optimistic that next years will bring significant advances. Therefore, I am in close contact with lattice and LCSR experts und look up frequently the updates by FLAG [18] and HFAG [26].

In the course of this lecture we will give several examples which demonstrate that it is very important to fulfil the requirements listed above if we want to reach the Zeptouniverse before a $100 \mathrm{TeV}$ collider will be built.

\section{Main Strategy}

\subsection{General View}

Let us then assume that the CKM parameters have been determined with high precision and non-perturbative parameters, relevant both for the SM and its extentions, have been calculated accurately. Having then precise SM predictions let us assume that future precise measurements of various observables have identified a number of deviations from SM predictions so that without any doubt we can conclude that some NP is at work. The question then arises what kind of NP could be responsible for these deviations.

Clearly, the most interesting and favourable situation, that one could hope for, would be a direct discovery of new particles at the LHC which would indicate at least first steps towards the Zeptouniverse. The interplay of LHC findings with quark and lepton flavour data and those on EDMs would be exciting and would teach us a lot but we cannot exclude at present that the lightest new particles are out of the reach of the LHC.

This would make the life of flavour physicists much harder but still it is our duty to develop efficient tools for the identifcation of NP through rare processes, that is through quantum fluctuations. As summarized in [1] this will require

- many precise measurements of many observables and precise theory,

- intensive studies of correlations between many observables in a given extension of the SM with the goal to identify patterns of deviations from the SM expectations characteristic for this extension, 
- intensive studies of correlations between low energy precision measurements, including electroweak precision tests and the measurements at the highest available energy, that is in the coming decades the measurements of a multitude of observables in proton-proton collisions at the LHC.

Now in the search for NP one distinguishes between bottom-up and top-down approaches. In my view both approaches should be persued but I think that in the context of flavour physics and simultaneous exploration of short distance physics, both through LHC and high precision experiments, the top-down approach is more powerful. I presented my arguments already in [2] but let me repeat them briefly here.

In the bottom-up approach one constructs effective field theories involving only light degrees of freedom including the top quark and Higgs boson in which the structure of the effective Lagrangians is governed by the symmetries of the SM and often other hypothetical symmetries. This approach is rather powerful in the case of electroweak precision studies and definitely teaches us something about $\Delta F=2$ transitions. In particular lower bounds on NP scales, depending on the Lorentz structure of involved operators, can be derived from the data [27-29]. However, except for the case of minimal flavour violation (MFV) and closely related approaches based on flavour symmetries, the bottom-up approach ceases, in my view, to be useful in $\Delta F=1$ decays. Indeed, in this case the appearnce of very many operators, that are allowed to enter the effective Lagrangians with coefficients that are basically unknown [30,31], lowers the predictive power of theory. In this approach then the correlations between various $\Delta F=2$ and $\Delta F=1$ observables in $K, D, B_{d}$ and $B_{s}$ systems are either not visible or very weak, again except MFV and closely related approaches. Moreover the correlations between flavour violation in low energy processes, electroweak precision observables and flavour violation in high energy processes are washed out. Again MFV is among few exceptions. The situation improves when only a certain class of processes, like $b \rightarrow s \mu^{+} \mu^{-}$and $b \rightarrow s \nu \bar{v}$, are considered and the invariance of NP under the full SM gauge symmetry $S U(3)_{c} \otimes S U(2)_{L} \otimes U(1)_{Y}$ is imposed. This was stressed in particular in [32] but also in [33,34]. Still, as we will discuss below, this approach has limitations in identifying the correct route to short distance scales.

On the other hand in the top-down approach one constructs first a specific model with heavy degrees of freedom. For high energy processes, where the energy scales are of the order of the masses of heavy particles one can directly use this "full theory" to calculate various processes in terms of the fundamental parameters of a given theory. For low energy processes one again constructs the low energy theory by integrating out heavy particles. The advantage over the bottomup approach is that now the Wilson coefficients of the resulting local operators are calculable in terms of the fundamental parameters of this theory. In this manner correlations between various observables belonging to different mesonic systems and correlations between low energy and highenergy observables and also electroweak precision tests are possible. Such correlations are less sensitive to free parameters than individual observables and represent patterns of flavour violation characteristic for a given theory. These correlations can in some models differ strikingly from the ones of the SM and of the MFV approach.

Having the latter strategy in mind I have in the last ten years investigated together with my young collaborators flavour violating and $\mathrm{CP}$-violating processes in a multitude of models. The 


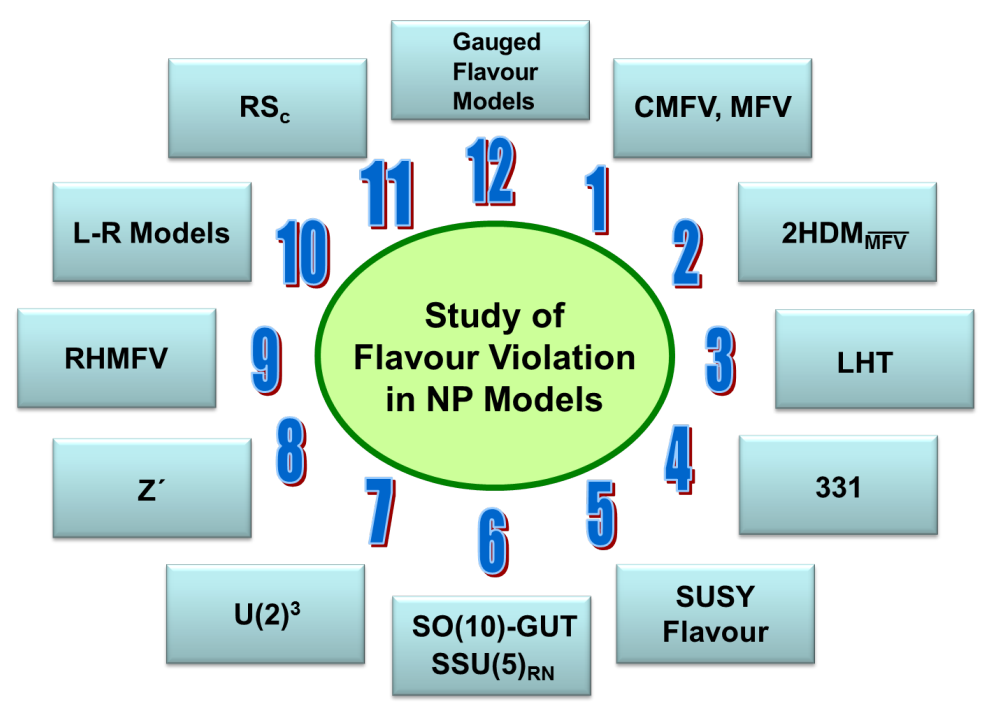

Figure 1: Studing multitude of extensions of the Standard Model.

names of models analyzed by us until now are collected in Fig. 1. A summary of these studies with brief descriptions of all these models can be found in $[1,35,36]$. Here, I want to summarize some of the lessons gained through these studies and subsequently concentrate on most recent analyses that have been performed by us in 2014 and 2015 .

\subsection{Superstars and Stars for 2015-2025 in Quark Flavour Physics}

Yet, before doing this it is useful to list the most promising observables in the search for NP. There are many measurements one can do and many observables one can calculate but in my view the following ones will lead the discussions and searches for NP in the coming ten years.

- $\Delta F=2$ observables:

$$
\Delta M_{s}, \quad \Delta M_{d}, \quad S_{\psi K_{S}} \quad S_{\psi \phi}, \quad \varepsilon_{K},
$$

with $S_{\psi K_{S}}$ and $S_{\psi \phi}$ being mixing induced CP-asymmetries that are measured in $B_{d}\left(\bar{B}_{d}\right)$ decays. In spite of non-leptonic nature of the relevant decays, these asymmetries are subject to only small hadronic uncertainties originating primarly in QCD penguin contributions. On the other hand it is expected that lattice QCD will provide in the next five years rather precise values for the hadronic matrix elements relevant for $\Delta M_{s}, \Delta M_{d}$ and $\varepsilon_{K}$ including the ones present in the extensions of the SM. The data on these three quantities are already very precise. The ones for $S_{\psi K_{S}}$ and $S_{\psi \phi}$ should be precise in the second half of this decade. Their present values are given in (4.6).

- Angular observables and branching ratios in the decays

$$
B \rightarrow K^{*} \ell^{+} \ell^{-}, \quad B \rightarrow K \ell^{+} \ell^{-}
$$


which are presently stars of flavour physics. In particular in the case of $B \rightarrow K^{*} \ell^{+} \ell^{-}$the presence of a multitude of angular observables could in principle offer detailed insight in the structure of NP. Yet there are still important issues of long distance uncertainties in $B \rightarrow$ $K^{*} \ell^{+} \ell^{-}$which remain to be clarified. The decays $B \rightarrow K \ell^{+} \ell^{-}$are theoretically cleaner and possible signs of the violation of lepton universality seen in the data are very intriguing.

- The branching ratios for

$$
\mathscr{B}\left(B_{s} \rightarrow \mu^{+} \mu^{-}\right), \quad \mathscr{B}\left(B_{d} \rightarrow \mu^{+} \mu^{-}\right) .
$$

The weak decay constants entering these branching ratios should be known in the coming years with the accuracy of $(1-2) \%$. The uncertainty in $\left|V_{t s}\right|$ and $\left|V_{t d}\right|$ can be totally removed within CMFV by relating these branching ratios to $\Delta M_{s}$ and $\Delta M_{d}$, respectively [37]. In other scenarios the corresponding relation does not fully remove this uncertainty but can reduce it significantly. The $\hat{B}_{s, d}$ parameters entering these relations should be known within $2 \%$ in this decade from lattice QCD.

- The branching ratios

$$
\mathscr{B}(B \rightarrow K v \bar{v}), \quad \mathscr{B}\left(B \rightarrow K^{*} v \bar{v}\right), \quad \mathscr{B}\left(B \rightarrow X_{s} v \bar{v}\right) .
$$

Even if these branching ratios are sensitive to form factor uncertainties, which should be decreased significantly in this decade, they are less subject to long distance effects present in $b \rightarrow s \mu^{+} \mu^{-}$. Moreover, they offer powerful means to study the effects of right-handed currents [34,38-42].

- The branching ratios for

$$
\mathscr{B}\left(K^{+} \rightarrow \pi^{+} v \bar{v}\right), \quad \mathscr{B}\left(K_{L} \rightarrow \pi^{0} v \bar{v}\right) .
$$

These two branching ratios are basically free from hadronic uncertainties and the ones in the charm contribution to $K^{+} \rightarrow \pi^{+} v \bar{v}$ can be reduced in the future through lattice QCD. The very strong dependence on $\left|V_{c b}\right|$ of both branching ratios and on $\left|V_{u b}\right|$ in the case of $K_{L} \rightarrow$ $\pi^{0} v \bar{v}$ will remain an issue for some time. A recent analysis in [23] demonstrates it explicitly. On the other hand the triple correlation between these two branching ratios and $S_{\psi K_{S}}$ within the SM and CMFV models is practically free from this dependence $[43,44]$. In this context one should mention the ratio $\varepsilon^{\prime} / \varepsilon$ which is very sensitive to NP effects. Unfortunately, the status of hadronic matrix elements relevant for the calculation of $\varepsilon^{\prime} / \varepsilon$ is far from being satisfactory. While the ones relevant for electroweak penguin contributions are known within the accuracy of 5\% from lattice QCD [45], the ones of QCD penguins are known only in the large $N$ limit of QCD [46]. From present perspective it is unlikely that $\varepsilon^{\prime} / \varepsilon$ will become the star of flavour physics in the coming years but could become it around 2020 and in the next decade.

$$
\mathscr{B}\left(B^{+} \rightarrow \tau^{+} v_{\tau}\right), \quad \mathscr{B}\left(B \rightarrow D \tau \nu_{\tau}\right) \quad \mathscr{B}\left(B \rightarrow D^{*} \tau \nu_{\tau}\right)
$$


which play important role in searching for NP effects mediated by charged scalars and gauge bosons $[47,48]$. Moreover, the present data from BABAR [49] on the last two show the largest departures from the SM in flavour physics. We refer to [50-56] for details.

\subsection{Superstars and Stars for 2015-2025 in Lepton Flavour Physics}

There is no question about that also lepton flavour physics will play a very important role in identifying NP beyond the SM. In particular the following decays should provide a deep insight into the dynamics at short distance scales:

- First of all the decays

$$
\mu \rightarrow e \gamma, \quad \tau \rightarrow e \gamma, \quad \tau \rightarrow \mu \gamma,
$$

that are governed by dipole operators. The improved bound on the first one from MEG [57]

$$
\mathscr{B}(\mu \rightarrow e \gamma) \leq(5.7) \times 10^{-13}
$$

puts significant constraints on the parameters of various extensions of the SM. The improvement on the other two decays is expected from Belle II and LHCb.

- Next come

$$
\mu^{-} \rightarrow e^{-} e^{+} e^{-}, \quad \tau^{-} \rightarrow \mu^{-} \mu^{+} \mu^{-}, \quad \tau^{-} \rightarrow e^{-} e^{+} e^{-} .
$$

These decays are very interesting as they are strongly correlated with $\mu \rightarrow e \gamma, \tau \rightarrow e \gamma$ and $\tau \rightarrow \mu \gamma$ and these correlations are different for different models.

- Also the four decays

$$
\tau^{-} \rightarrow e^{-} \mu^{+} e^{-}, \quad \tau^{-} \rightarrow \mu^{-} e^{+} \mu^{-},
$$

and

$$
\tau^{-} \rightarrow \mu^{-} e^{+} e^{-}, \quad \tau^{-} \rightarrow e^{-} \mu^{+} \mu^{-}
$$

will enrich the search for NP.

- $\mu-e$ conversion in nuclei, even if subject to hadronic uncertainties, could become the star of lepton flavour physics at the end of this decade. The dedicated J-PARC experiment PRISM/PRIME should reach a sensitivity of $\mathscr{O}\left(10^{-18}\right)$ [58]. Also semi-leptonic $\tau$ decays like $\tau \rightarrow \pi \mu$ e should not be forgotten.

For further detailed review of LFV see [59-61]. An experimenter's guide for charged LFV can be found in [62].

\subsection{Interplay of Quark and Lepton Flavour Violation}

Of special interest are decays that proceed through both quark flavour and lepton flavour violating transitions. These are in particular

$$
K_{L, S} \rightarrow \mu e, \quad K_{L, S} \rightarrow \pi^{0} \mu e
$$

and

$$
B_{d, s} \rightarrow \mu e, \quad B_{d, s} \rightarrow \tau e \quad B_{d, s} \rightarrow \tau \mu .
$$

A natural mechanism responsible for such transitions are tree-level exchanges of leptoquarks. But they can also be generated at one-loop level, an example being LHT model [63]. 


\subsection{Electric Dipole Moments and $(g-2)_{e, \mu}$}

Even if these observables are flavour conserving they put strong bounds on extensions of the SM. The $(g-2)_{\mu}$ anomaly found at Brookhaven should be clarified by Fermilab at the end of this decade. A recent review about EDMs can be found in [64] which updates the review in [65]. See also [66].

After this collection of most important decays let us enter some details.

\section{The Power of Correlations between Flavour Observables}

\subsection{Preliminaries}

In studying correlations between various decays it is important to remember that [67]

- Correlations between decays of different mesons test the flavour structure of couplings or generally flavour symmeteries.

- Correlations between decays of a given meson test the Dirac structure of couplings.

We will first look at the first correlations by comparing those within MFV models based on $U(3)^{3}$ flavour symmetry with the ones present in models with $U(2)^{3}$ flavour symmetry. To this end we will assume that in the latter case, similar to MFV, only the left-handed couplings are relevant.

\subsection{CMFV and MFV}

These models are based on flavour $U(3)^{3}$ symmetry and their most striking predictions are:

- No new sources of flavour and CP violation (excluding flavour blind phases) imply ${ }^{3}$.

$$
S_{\psi K_{S}}=\sin 2 \beta, \quad S_{\psi \phi}=S_{\psi \phi}^{\mathrm{SM}}=0.036 \pm 0.002
$$

- Stringent correlations between $K, B_{d}$ and $B_{s}$ systems and in particular between $\Delta F=2$ and $\Delta F=1$ observables.

- For fixed CKM parameters determined in tree-level decays, $\left|\varepsilon_{K}\right|, \Delta M_{s}$ and $\Delta M_{d}$, if modified, can only be enhanced relative to SM predictions [68]. Moreover this happens in a correlated manner [36,69]. The implications of this property are rather powerful. Finding in the future SM prediction for one of these three observables above its experimental value, will signal the presence of non-CMFV interactions.

- Absence of right-handed charged currents.

Let me recall some of these correlations as they could be soon relevant. The first two are the ones in models with constrained Minimal Flavout Violation (CMFV) [70,71]

$$
\frac{\mathscr{B}\left(B_{s} \rightarrow \mu^{+} \mu^{-}\right)}{\mathscr{B}\left(B_{d} \rightarrow \mu^{+} \mu^{-}\right)}=\frac{\tau\left(B_{s}\right)}{\tau\left(B_{d}\right)} \frac{m_{B_{s}}}{m_{B_{d}}} \frac{F_{B_{s}}^{2}}{F_{B_{d}}^{2}}\left|\frac{V_{t s}}{V_{t d}}\right|^{2},
$$

\footnotetext{
${ }^{3}$ Our definition of $S_{\psi \phi}$ differs by sign from the one used by LHCb and HFAG.
} 


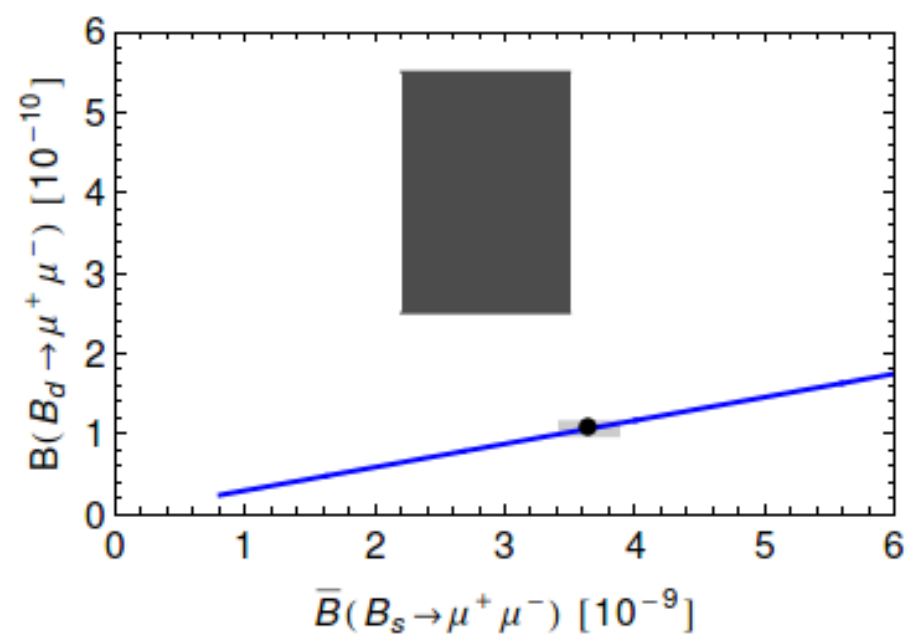

Figure 2: $\mathscr{B}\left(B_{d} \rightarrow \mu^{+} \mu^{-}\right)$vs $\overline{\mathscr{B}}\left(B_{s} \rightarrow \mu^{+} \mu^{-}\right)$in models with $C M F V$. SM is represented by the light grey area with black dot. Dark gray region: Overlap of exp $1 \sigma$ ranges for $\overline{\mathscr{B}}\left(B_{s} \rightarrow \mu^{+} \mu^{-}\right)=(2.8 \pm 0.7) \cdot 10^{-9}$ and $\mathscr{B}\left(B_{d} \rightarrow \mu^{+} \mu^{-}\right)=\left(3.9_{-1.4}^{+1.6}\right) \times 10^{-10}$. Update of [1].

and $[37]^{4}$

$$
\frac{\mathscr{B}\left(B_{s} \rightarrow \mu^{+} \mu^{-}\right)}{\mathscr{B}\left(B_{d} \rightarrow \mu^{+} \mu^{-}\right)}=r \frac{\hat{B}_{d}}{\hat{B}_{s}} \frac{\tau\left(B_{s}\right)}{\tau\left(B_{d}\right)} \frac{\Delta M_{s}}{\Delta M_{d}}=r(34.5 \pm 0.8), \quad \frac{\hat{B}_{d}}{\hat{B}_{s}}=0.99 \pm 0.02
$$

where the departure of $r$ from unity measures effects which go beyond CMFV. This golden relation between $\Delta M_{s, d}$ and $B_{s, d} \rightarrow \mu^{+} \mu^{-}$does not involve $F_{B_{q}}$ and CKM parameters and consequently contains smaller hadronic and parametric uncertainties than (4.2). It involves only measurable quantities except for the ratio $\hat{B}_{s} / \hat{B}_{d}$ that is known from lattice calculations with impressive accuracy of roughly $\pm 2 \%$ [76] as given in (4.3). Consequently the r.h.s of this equation is already rather precisely known and this precision will be improved within this decade. This would allow us to identify possible NP in $B_{s, d} \rightarrow \mu^{+} \mu^{-}$decays and also in $\Delta M_{s, d}$ even if it was only at the level of $20 \%$ of the SM contributions. This is rather unique in the quark flavour physics and only the decays $K^{+} \rightarrow \pi^{+} v \bar{v}$ and $K_{L} \rightarrow \pi^{0} v \bar{v}$ can compete with this precision.

In fact, in the case of the latter decays one can find a theoretically clean relation between the branching ratios for $K^{+} \rightarrow \pi^{+} v \bar{v}$ and $K_{L} \rightarrow \pi^{0} v \bar{v}$ and $\sin 2 \beta$ that practically does not depend on CKM uncertainties, in particular $\left|V_{c b}\right|,\left|V_{u b}\right|$ and $\gamma$, even if the individual branching ratios for these decays are rather sensitive to the values of these parameters as stressed recently in [23]. We refer to $[43,44]$, Section 8 in this lecture and [23] for details.

The most recent data on $B_{s} \rightarrow \mu^{+} \mu^{-}$from LHCb and CMS collaborations give first indications that NP contributions to $B_{s} \rightarrow \mu^{+} \mu^{-}$are much smaller than the SM contribution itself. On the other hand the data on $B_{d} \rightarrow \mu^{+} \mu^{-}$exhibit some departure from SM expectations but we have to wait for improved data in order to see whether NP is at work here. We compare the relation (4.3) with present data in Fig. 2, where we included $\Delta \Gamma_{s}$ effects in $B_{s} \rightarrow \mu^{+} \mu^{-}$.

\footnotetext{
${ }^{4}$ As emphasized in [72] the dependence of the ratio of branching ratios in (4.2) on the elements of the CKM is more general than CMFV and applies to MFV at large [73-75].
} 
The most recent prediction in the SM that includes NNLO QCD corrections [77] and NLO electroweak corrections [78], put together in [79], and the most recent averages from the combined analysis of CMS and LHCb [80] are given as follows:

$$
\begin{gathered}
\overline{\mathscr{B}}\left(B_{s} \rightarrow \mu^{+} \mu^{-}\right)_{\mathrm{SM}}=(3.65 \pm 0.23) \cdot 10^{-9}, \quad \overline{\mathscr{B}}\left(B_{s} \rightarrow \mu^{+} \mu^{-}\right)=\left(2.8_{-0.6}^{+0.7}\right) \times 10^{-9}, \\
\mathscr{B}\left(B_{d} \rightarrow \mu^{+} \mu^{-}\right)_{\mathrm{SM}}=(1.06 \pm 0.09) \times 10^{-10}, \quad \mathscr{B}\left(B_{d} \rightarrow \mu^{+} \mu^{-}\right)=\left(3.9_{-1.4}^{+1.6}\right) \times 10^{-10}
\end{gathered}
$$

The "bar" in the case of $B_{s} \rightarrow \mu^{+} \mu^{-}$indicates that $\Delta \Gamma_{s}$ effects [81-83] have been taken into account.

Clearly in the case of $B_{d} \rightarrow \mu^{+} \mu^{-}$large deviations from SM prediction are still possible. But in the case of $\overline{\mathscr{B}}\left(B_{s} \rightarrow \mu^{+} \mu^{-}\right)$deviations by more than $30 \%$ from its $\mathrm{SM}$ value seem rather unlikely. Yet, the reduction of the error in the SM prediction down to $3-4 \%$ is still possible and this would allow to see NP at the level of $20 \%$ provided the measurements improve.

We observe that the data for $\overline{\mathscr{B}}\left(B_{s} \rightarrow \mu^{+} \mu^{-}\right)$are by $1.2 \sigma$ lower than the SM prediction. Yet, at this stage I would like to express one warning. The authors in [79] used the inclusive value for $\left|V_{c b}\right|$ in obtaining quoted result. If they had used the exclusive one, the central value for the branching ratio would move down to $3.1 \times 10^{-9}$, that fully overlaps with the data.

In CMFV [70] and MFV at large [75], that are both based on the $U(3)^{3}$ flavour symmetry, the measurement of the mixing induced asymmetry $S_{\psi K_{S}}$ together with the unitarity of the CKM implies that the analogous asymmetry in the $B_{s}^{0}-\bar{B}_{s}^{0}$ system, $S_{\psi \phi}$, is very small. See (4.1). Presently the data give

$$
S_{\psi K_{S}}=0.679 \pm 0.020, \quad S_{\psi \phi}=0.010 \pm 0.039
$$

where the first number comes from PDG and the second from the most recent analysis of the LHCb [84] which dominates this determination. Although $S_{\psi \phi}$ is found to be small it could still significantly differ from its SM value, in particular if it had negative sign. We are looking forward to new world averages of these asymmetries in the coming years.

Now, the SM faces the following problem. In order to reproduce the data on $S_{\psi K_{S}}$ the value of $\left|V_{u b}\right|$ has to be close to its exclusive determination but then $\varepsilon_{K}$ turns out to be too small to agree with very precise data [4]. The solution to this problem is a large value of $\left|V_{c b}\right|$ in the ballpark of its inclusive determination but this shifts, with present lattice input, the SM values of $\Delta M_{s}$ and $\Delta M_{d}$ above the data. Going beyond the SM but staying withing CMFV allows to improve the agreement of $\varepsilon_{K}$ with the data by increasing the box function $S$ above its $\mathrm{SM}$ value. This is natural within CMFV models as stated above: $S$ and $\varepsilon_{K}$ can only be increased [68]. But this function also enters $\Delta M_{s}$ and $\Delta M_{d}$ and they are again increased above their experimental values. As analyzed in detail in [85] only for specific values of non-perturbative parameters entering $\Delta M_{s, d}$ can SM and CMFV be saved.

In fact this pattern is fully consistent with the most recent UTfitters result in [86] where the determined values of their coefficients $C_{B_{d}}=0.81 \pm 0.12$ and $C_{B_{s}}=0.87 \pm 0.09$, while consistent with unity as obtained within the SM, indicate that the data favour NP that suppresses $\Delta M_{s, d}$ which is impossible within CMFV [68]. In summary, it appears as the correlation between $\varepsilon_{K}, \Delta M_{s, d}$ and $S_{\psi K_{S}}$ could turn out to be a problem for SM and CMFV but clear cut conclusions can only be reached when the precision on lattice QCD calculations improves. 


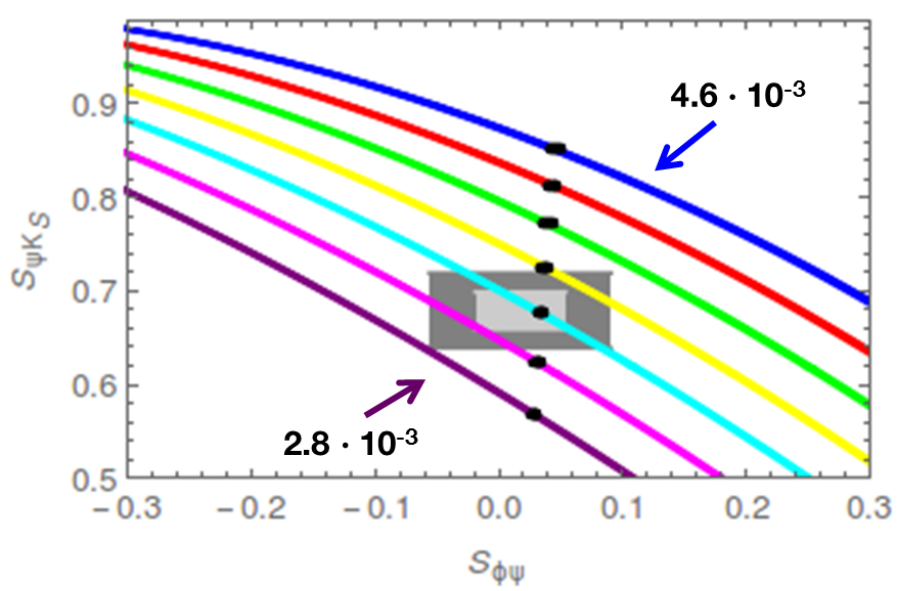

Figure 3: $S_{\psi K_{S}}$ vs. $S_{\psi \phi}$ in models with $U(2)^{3}$ symmetry for different values of $\left|V_{u b}\right|$ and $\gamma \in\left[58^{\circ}, 78^{\circ}\right]$. From top to bottom: $\left|V_{u b}\right|=0.0046$ (blue), 0.0043 (red), 0.0040 (green), 0.0037 (yellow), 0.0034 (cyan), 0.0031 (magenta), 0.0028 (purple). Light/dark gray: experimental $1 \sigma / 2 \sigma$ region. Update of [87].

On the other hand if one day the value of $\left|V_{u b}\right|$ above 0.0040 will be the correct one then the predicted value of $S_{\psi K_{S}}$ will be above the data and without new CP-violating phases we will not be able to reproduce its value in (4.6) [3].

My personal expectations are that the exclusive determinations of $\left|V_{u b}\right|$ and $\left|V_{c b}\right|$ will win and that NP in $\varepsilon_{K}$ but not $S_{\psi K_{S}}$ will be required. While in this case CMFV or MFV will still remain to be a valid framework, their future will depend on the precise values of hadronic matrix elements entering $\Delta M_{s}$ and $\Delta M_{d}$ and on other observables listed previously.

\section{$4.3 U(2)^{3}$ Symmetry}

The tensions between $\varepsilon_{K}$ and $\Delta M_{s, d}$ on one hand and between $\varepsilon_{K}$ and $S_{\psi K_{S}}$ on the other hand originate in the strict correlations between $K, B_{d}$ and $B_{s}$ system present in the SM and CMFV models. One elegant solution to this problem is the reduction of the flavour symmetry down to $U(2)^{3}[88-90]$. In this case the flavour symmetry is only between two light quark generations with the following important modifications relative to the $U(3)^{3}$ case:

- The formulae in (4.1) are modified to

$$
S_{\psi K_{S}}=\sin \left(2 \beta+\varphi^{\text {new }}\right), \quad S_{\psi \phi}=\sin \left(2\left|\beta_{S}\right|-\varphi^{\text {new }}\right),
$$

where $\beta_{s}=-1^{\circ}$ is up to the sign the phase of $-V_{t s}$ and $\varphi^{\text {new }}$ is a new phase.

- While the correlations between $\Delta M_{s}$ and $\Delta M_{d}$ and the relation in (4.3) remains true, the correlation between $\varepsilon_{K}$ and $\Delta M_{s, d}$ is broken.

Therefore, as seen in the plots in [91], the tensions discussed above are avoided in this NP scenario but as pointed out in [87] in the simplest versions of these models in which this symmetry is broken minimally, there is a stringent triple correlation $S_{\psi K_{S}}-S_{\psi \phi}-\left|V_{u b}\right|$ that constitutes an important test of these models. We show this correlation in Fig. 3 for $\gamma$ between $58^{\circ}$ and $78^{\circ}$. 
The latter dependence is very weak and is represented by the thickness of the lines. Note that in a $U(2)^{3}$ symmetric world, $\left|V_{u b}\right|$ could be determined with very small hadronic uncertainties by simply measuring $S_{\psi \phi}$ and $S_{\psi K_{S}}$. However, it is more interesting to extract $\left|V_{u b}\right|$ from tree level decays and check whether this triple correlation is respected by nature.

While at the time of the analysis in [87] still significant departures of $S_{\psi \phi}$ from its SM value were allowed, the improved data on this asymmetry indicate that the $\left|V_{u b}\right| \approx(3.4 \pm 0.3) \times 10^{-3}$ is favoured. This value is in perfect agreement with the most recent exclusive determinations of $\left|V_{u b}\right|$. Whether models with $U(2)^{3}$ symmetry will get some problems here depends on the future determinations of the three quantities in question.

But there is still another important point. In this simple scenario the relation (4.3) is still valid [87] even if the branching ratios and $\Delta M_{s, d}$ can all differ from their SM values. This means that if the experimental grey area in Fig. 2 will not move in the future but will decrease in size, the breakdown of $U(2)^{3}$ symmetry has to be either more involved or we have to look for other alternatives for NP. This brings us to a more general study of correlations between flavour observables.

\section{Correlations between Flavour Observables in Models with Tree Level FCNCs}

\subsection{Generalities}

During the last two years we have performed general analyses of flavour observables in models in which FCNC processes are mediated at tree-level by neutral gauge bosons $[41,92,93]$ and neutral scalars or pseudoscalars $[94,95]$. In addition we have made detailed analyses of FCNCs within the 3-3-1 models [96-98], in particular in view of the $B_{d} \rightarrow K^{*} \mu^{+} \mu^{-}$anomalies and the new experimental results on $B_{s} \rightarrow \mu^{+} \mu^{-}$[80]. A review of these analyses can be found in [1,2], where also the references to other papers written in 2013 and 2014 can be found. Our main goal in these papers was to identify correlations between several flavour observables that have not been presented in the past. Here I will mainly concentrate on our analyses done in 2014. But first let me recall why the correlations in $Z^{\prime}$ models are very instructive. The point is that the structure of such NP contributions is simple. Indeed, a tree level contribution to a $\Delta F=2$ transition, like particle-antiparticle mixing, mediated by a gauge boson $Z^{\prime}$ is described by the amplitude

$$
\mathscr{A}(\Delta F=2)=a \bar{\Delta}_{B}^{i j}\left(Z^{\prime}\right) \bar{\Delta}_{C}^{i j}\left(Z^{\prime}\right), \quad \bar{\Delta}_{B}^{i j}\left(Z^{\prime}\right)=\frac{\Delta_{B}^{i j}\left(Z^{\prime}\right)}{M_{Z^{\prime}}}
$$

where $\Delta_{B, C}^{i j}$ with $(B, C)=(L, R)$ are left-handed or right-handed couplings of $Z^{\prime}$ to quarks with $(i, j)$ equal to $(s, d),(b, d)$ and $(b, s)$ for $K^{0}, B_{d}^{0}$ and $B_{s}^{0}$ meson system, respectively. The overall flavour independent factor $a$ is a numerical constant that generally depends on $L$ and $R$ but we suppress this dependence. If we assume that only left-handed or right-handed couplings are present or that left-handed and right-handed couplings are either equal to each other or differ by sign, then this amplitude for a fixed $(i, j)$ is described by only two parameters, the magnitude and the phase of the reduced coupling $\bar{\Delta}_{B}^{i j}$.

On the other hand a tree-level amplitude for a $\Delta F=1$ transition like a leptonic or semi-leptonic decay of a meson with $\mu \bar{\mu}$ or $v \bar{v}$ appearing in the final state has the structure

$$
\mathscr{A}(\Delta F=1)=b \bar{\Delta}_{B}^{i j}\left(Z^{\prime}\right) \bar{\Delta}_{D}^{\mu \bar{\mu}}\left(Z^{\prime}\right), \quad \bar{\Delta}_{D}^{\mu \bar{\mu}}\left(Z^{\prime}\right)=\frac{\Delta_{D}^{\mu \bar{\mu}}\left(Z^{\prime}\right)}{M_{Z^{\prime}}}
$$


with $\bar{\Delta}_{B}^{i j}\left(Z^{\prime}\right)$ being the same quark couplings as in (5.1) and $b$ is again an overall factor. $D=(A, V)$ distinguishes between axial-vector and vector coupling to muons. For $v \bar{v}$ the couplings are chosen to be left-handed: $\Delta_{L}^{v \bar{v}}\left(Z^{\prime}\right)$. Clearly the same formulae with different values of couplings and the factors $a$ and $b$ apply to a tree-level exchange of $Z$, a heavy pseudoscalar $A$ and a heavy scalar $H$.

Now we can constrain the $\Delta_{B}^{b s}\left(Z^{\prime}\right)$ couplings by the data on $\Delta M_{s}$ and the CP-asymmetry $S_{\psi \phi}$ and the couplings $\Delta_{B}^{b d}\left(Z^{\prime}\right)$ by the data on $\Delta M_{d}$ and the CP-asymmetry $S_{\psi K_{S}}$. In the case of $\Delta_{B}^{s d}\left(Z^{\prime}\right)$ we have mainly $\varepsilon_{K}$ to our disposal as $\Delta M_{K}$ being subject to significant hadronic uncertainties provides much weaker constraint than $\varepsilon_{K}$ in the models in question.

Once these constraints on the magnitude and the phase of new couplings are imposed and the allowed values are used for the predictions for rare decays it is evident that correlations between various observables are present, although the correlations between the decays with $v \bar{v}$ and those with $\mu^{+} \mu^{-}$in the final state require more information, except FCNCs mediated by $Z$ boson, where these couplings are known. More about it later.

It is particularly interesting that the pattern of these correlations depends on whether a gauge boson, a scalar or pseudoscalar mediates the FCNC transition. As the scalar contributions cannot interfere with SM contributions, only enhancements of branching ratios are possible in this case. A tree-level gauge boson contribution and pseudoscalar contribution interfer generally with the SM contribution but the resulting correlations between observables have different pattern because of the $i$ in the coupling $i \gamma_{5}$ of a pseudoscalar to leptons. Detailed analytic explanations of these differences and the corresponding plots can be found in [95].

\subsection{Left-handed and Right-handed Couplings in Tree-Level FCNCs}

$\Delta F=2$ transitions provide a useful information about short distance dynamics and played already very important role in constraining the extensions of the SM in the last thirty years. Yet, I would like to stress one limitation of such tests of NP. Consider a contribution from a tree-level heavy neutral gauge boson to $\varepsilon_{K}$. Assuming that both left-handed and right-handed couplings to quarks are involved, the general structure of this contribution is as follows:

$$
\Delta \varepsilon_{K}=\operatorname{Im}\left(a g_{L}^{2}+a g_{R}^{2}+b g_{L} g_{R}\right)
$$

where $a$ and $b$ are real and the couplings $g_{L}$ and $g_{R}$ can be complex. Note the equality of the coefficients in the first two terms which follows form the vectorial structure of QCD interactions. Indeed the coefficients $a$ and $b$ encode the information about hadronic matrix elements and QCD effects, in particular renormalization group effects. Typically $b \approx 150 a$. Analogous formulae can be written for $\Delta M_{s, d}$ but in this case $b \approx 7 a$.

As the expression in (5.3) is symmetric under the interchange of left and right, even if $\Delta \varepsilon_{K}$ will indeed be found experimentally to be non-zero, it will not be possible to decide on the basis of $\varepsilon_{K}$ alone whether left-handed couplings or right-handed couplings or both couplings are responsible for this signal of NP. The same comment applies to $\Delta M_{s, d}$. But the case of $\Delta F=1$ transitions, in particular of rare $K$ and rare $B_{s, d}$ decays, is different and they are crucial for getting deeper insight into the structure of the dynamics at very short distance scales.

Indded, let us consider a number of prominent rare decays and divide them into two classes:

Class A: Decays that are governed by vector $\left(V=\gamma_{\mu}\right)$ quark couplings. These are for instance

$$
K^{+} \rightarrow \pi^{+} v \bar{v}, \quad K_{L} \rightarrow \pi^{0} v \bar{v}, \quad B \rightarrow K \nu \bar{v}, \quad B \rightarrow K \mu^{+} \mu^{-} .
$$


In this case the change from left-handed to right-handed quark couplings does not introduce any change of the sign of NP contribution relatively to the SM one.

Class B: Decays that are governed by axial-vector $\left(A=\gamma_{\mu} \gamma_{5}\right)$ quark couplings. These are for instance

$$
K_{L} \rightarrow \mu^{+} \mu^{-}, \quad B \rightarrow K^{*} v \bar{v}, \quad B_{s, d} \rightarrow \mu^{+} \mu^{-}, \quad B_{d} \rightarrow K^{*} \mu^{+} \mu^{-} .
$$

In this case the change from left-handed to right-handed couplings implies the sign flip of NP contribution relatively to the SM one. Strictly speaking in the case of $B \rightarrow K^{*} v \bar{v}$ and $B_{d} \rightarrow K^{*} \mu^{+} \mu^{-}$ this rule only applies if the contributions from the longitudinal and parallel transversity components dominate. For perpendicular component there is no sign flip.

Thus if there is a correlation between two observables belonging to class A and B in the presence of left-handed couplings, it is changed into anti-correlation when right-handed couplings are at work. This difference allows then to probe whether one deals with left-handed or right-handed couplings. Of course if both left-handed and right-handed couplings are involved the structure of correlations is modified but still studying it one can in principle extract the relative size of these couplings from the data. Moreover, if there is a correlation or anti-correlation of two observables belonging to one class, the flip of sign of $\gamma_{5}$ will not have an impact on these relations but can of course have an impact on whether a given observable is suppressed or enhanced relative to the SM prediction. A graphical representation of these properties are the DNA charts [1] which we will briefly discuss now.

\section{Towards the New SM with the Help of DNA-Charts}

The identification of NP indirectly will require many measurements. Fortunately the coming years should provide important experimental input for this goal. In Fig. 4 (slightly different from the one in [1]) we collect those processes which we think will play the dominant role in testing the short distance structure during the second run of the LHC and in the search for NP beyond the LHC reach. In addition to $\Delta F=2$ processes and rare $K$ and $B_{s, d}$ decays we added other measurements not related to quark physics that we already mentioned previously. In fact simultaneous study of the outcome of direct searches for NP at the LHC, electroweak precision tests of BSM models suggested for the explanations of anomalies in the quark sector, charged lepton flavour violation, EDMs and $(g-2)_{\mu, e}$ could indeed help us in the identification of the correct route to the Zeptouniverse and allow us to construct a more fundamental theory than the SM.

But as far as quark physics is concerned very important are future precise determinations of CKM parameters and those of non-perturbative parameters from lattice QCD. This would allow us to reduce or even remove the main uncertainties that are present in the SM predictions for various FCNC observables. In fact as shown in $[85,93]$ in explicit terms the pattern of NP required for the explanations of deviations from SM predictions depends crucially on this input.

Once the CKM and hadronic parameters are sufficiently known, the search for NP becomes easier as now SM predictions are precise and serve as candles of flavour physics allowing us to see whether there is something beyond the dynamics we know. In this spirit, as emphasized in [1], already the pattern of signs of departures from SM expectations in various observables and the correlations or anti-correlations between these departures could exclude or support certain NP 


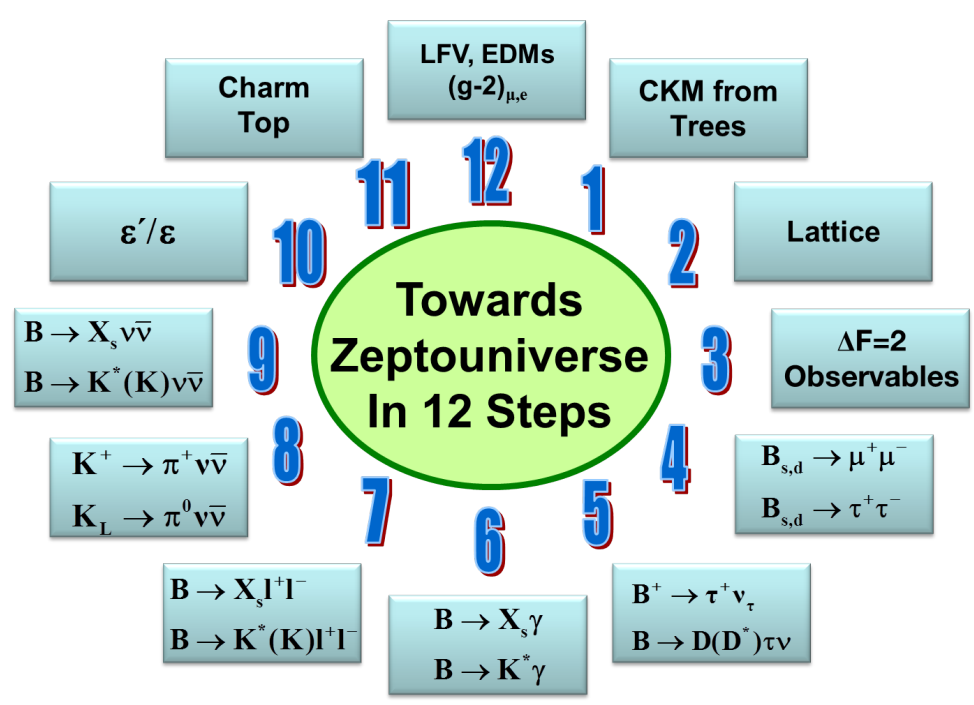

Figure 4: Towards the Zeptouniverse in 12 Steps.

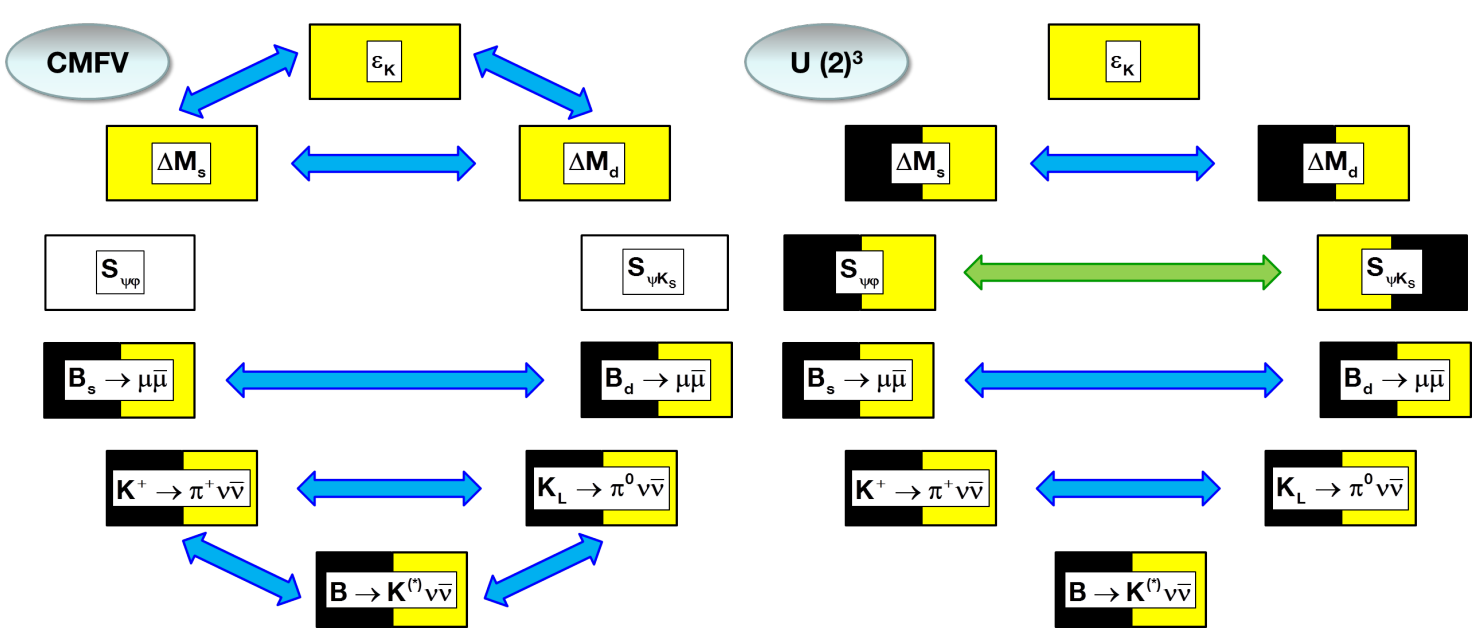

Figure 5: DNA-chart of MFV models (left) and of $U(2)^{3}$ models (right). Yellow means enhancement, black means suppression and white means no change. Blue arrows $\Leftrightarrow$ indicate correlation and green arrows $\Leftrightarrow$ indicate anti-correlation. From [1].

scenarios. In order to depict various possibilities in a transparent manner we have proposed a DNAchart to be applied separately to each NP scenario. In Fig. 5 we show the DNA-chart of MFV and the corresponding chart for $U(2)^{3}$ models. The DNA-charts representing models with left-handed and right-handed flavour violating couplings of $Z$ and $Z^{\prime}$ can be found in Fig. 6.

One can check that these charts summarize compactly the (anti-) correlations between processes of class $\mathrm{A}$ and $\mathrm{B}$, that we discussed before and also the correlations and anti-correlations within each class. In particular the change of a correlation into an anti-correlation between two observables belonging to two different classes, when left-handed couplings are changed to right- 


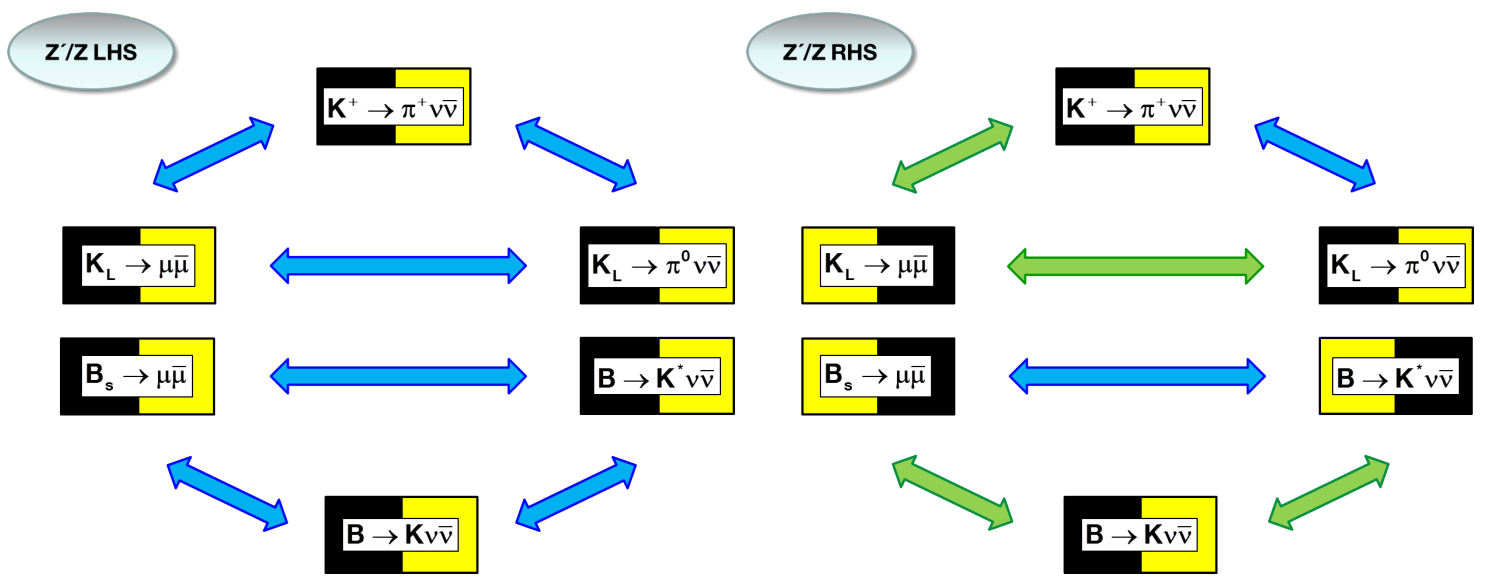

Figure 6: DNA-charts of $Z^{\prime}$ models with LH and RH currents. Yellow means enhancement, black means suppression and white means no change. Blue arrows $\Leftrightarrow$ indicate correlation and green arrows $\Leftrightarrow$ indicate anti-correlation. From [1].

handed ones, are clearly visible in these charts. We observe the following features:

- Comparing the DNA-charts of CMFV and $U(2)^{3}$ models in Fig. 5 we observe that the correlations between $K$ and $B_{s, d}$ systems are broken in the $U(2)^{3}$ case as the flavour symmetry is reduced from $U(3)^{3}$ down to $U(2)^{3}$. The anti-correlation between $S_{\psi \phi}$ and $S_{\psi K_{S}}$ is just the one shown in Fig. 3.

- As the decays $K^{+} \rightarrow \pi^{+} v \bar{v}, K_{L} \rightarrow \pi^{0} v \bar{v}$ and $B \rightarrow K v \bar{v}$ belonging to class A are only sensitive to the vector quark currents, they do not change when the couplings are changed from left-handed to right-handed ones. On the other hand the remaining three decays in Fig. 6 belonging to class $\mathrm{B}$ are sensitive to axial-vector couplings implying interchange of enhancements and suppressions when going from $L$ to $R$ and also change of correlations to anti-correlations between the latter three and the former three decays. Note that the correlation between $B_{s} \rightarrow \mu^{+} \mu^{-}$and $B \rightarrow K^{*} \mu^{+} \mu^{-}$does not change as both decays are sensitive only to axial-vector coupling if in the latter case the contribution from the longitudinal and parallel transversity components dominate.

- However, it should be remarked that in order to obtain the correlations or anti-correlations in LHS and RHS scenarios it was assumed in the DNA charts presented here that the signs of the left-handed couplings to neutrinos and the axial-vector couplings to muons are the same which does not have to be the case. If they are opposite the correlations between the decays with neutrinos and muons in the final state change to anti-correlations and vice versa.

- On the other hand due to $S U(2)_{L}$ symmetry the left-handed $Z^{\prime}$ couplings to muons and neutrinos are equal and this implies the relation

$$
\Delta_{L}^{v \bar{v}}\left(Z^{\prime}\right)=\frac{\Delta_{V}^{\mu \bar{\mu}}\left(Z^{\prime}\right)-\Delta_{A}^{\mu \bar{\mu}}\left(Z^{\prime}\right)}{2} .
$$

Therefore, once two of these couplings are determined the third follows uniquely without the freedom mentioned in the previous item. 
- In the context of the DNA-charts in Fig. 6, the correlations involving $K_{L} \rightarrow \pi^{0} v \bar{v}$ apply only if NP contributions carry some CP-phases. If this is not the case the branching ratio for $K_{L} \rightarrow \pi^{0} v \bar{v}$ will remain unchanged relativ to the SM one.

If in the case of tree-level $Z^{\prime}$ and $Z$ exchanges both LH and RH quark couplings are present and are equal to each other (LRS scenario) or differ by sign (ALRS scenario), then one finds [41]

- In LRS NP contributions to $B_{s, d} \rightarrow \mu^{+} \mu^{-}$vanish but they are present in $K_{L} \rightarrow \pi^{0} v \bar{v}, K^{+} \rightarrow$ $\pi^{+} v \bar{v}, B_{d} \rightarrow K \mu^{+} \mu^{-}$and $B \rightarrow K v \bar{v}$.

- In ALRS NP contributions to $B_{s, d} \rightarrow \mu^{+} \mu^{-}$are non-vanishing. On the other hand they are absent in the case of $K_{L} \rightarrow \pi^{0} v \bar{v}, K^{+} \rightarrow \pi^{+} v \bar{v}, B_{d} \rightarrow K \mu^{+} \mu^{-}$and $B \rightarrow K v \bar{v}$.

- In $B_{d} \rightarrow K^{*} \mu^{+} \mu^{-}$and $B \rightarrow K^{*} v \bar{v}$ this rule is more complicated as already stated above but generally the LH and RH contributions interfere destructively in LRS and constructively in ALRS. The details depend on form factors.

\section{Adressing Anomalies in $b \rightarrow s \ell^{+} \ell^{-}$Transitions}

\subsection{General Discussion}

We will next address the first anomalies listed in the overture.

In addition to anomalies in angular observables in $B_{d} \rightarrow K^{*} \mu^{+} \mu^{-}$found by LHCb at low $q^{2}$ also some departures from the SM at large $q^{2}$ in the ratios

$$
\mathscr{R}_{K \mu \mu}=\frac{\mathscr{B}\left(B^{+} \rightarrow K^{+} \mu^{+} \mu^{-}\right)^{[15,22]}}{\mathscr{B}\left(B^{+} \rightarrow K^{+} \mu^{+} \mu^{-}\right)_{\mathrm{SM}}^{[15,22]}}, \quad \mathscr{R}_{K^{*} \mu \mu}=\frac{\mathscr{B}\left(B^{0} \rightarrow K^{* 0} \mu^{+} \mu^{-}\right)^{[15,19]}}{\mathscr{B}\left(B^{0} \rightarrow K^{* 0} \mu^{+} \mu^{-}\right)_{\mathrm{SM}}^{[15,19]}}
$$

are found. Here the superscripts refer to the range in $q^{2}$ in $\mathrm{GeV}^{2}$. Both ratios are below unity as we will see in the plots below. Considering large $q^{2}$ allows to suppress dipole operators contributions and make the correlations with $B_{s} \rightarrow \mu^{+} \mu^{-}$and $b \rightarrow s v \bar{v}$ transitions more transparent.

To this end we define the ratios

$$
\begin{gathered}
\mathscr{R}_{\mu \mu}=\frac{\mathscr{B}\left(B_{s} \rightarrow \mu^{+} \mu^{-}\right)}{\mathscr{B}\left(B_{S} \rightarrow \mu^{+} \mu^{-}\right)_{\mathrm{SM}}}, \\
\mathscr{R}_{K}=\frac{\mathscr{B}(B \rightarrow K v \bar{v})}{\mathscr{B}\left((B \rightarrow K v \bar{v})_{\mathrm{SM}}\right.}, \quad \mathscr{R}_{K^{*}}=\frac{\mathscr{B}\left(B \rightarrow K^{*} v \bar{v}\right)}{\mathscr{B}\left(\left(B \rightarrow K^{*} v \bar{v}\right)_{\mathrm{SM}}\right.} .
\end{gathered}
$$

Interestingly the present data can be summarized by

$$
\mathscr{R}_{\mu \mu} \approx 0.80, \quad \mathscr{R}_{K \mu \mu} \approx 0.90, \quad \mathscr{R}_{K^{*} \mu \mu} \approx 0.75
$$

The important point is that they all are significantly below unity.

As shown in recent papers [33,99-102] these anomalies and in particular those at low $q^{2}$ can be reproduced by the shifts in the Wilson coefficients $C_{9}$ and $C_{10}$ with

$$
C_{9}^{\mathrm{NP}} \approx-C_{10}^{\mathrm{NP}} \approx-0.6
$$


The precise values depending on the paper. The solution with NP being present only in $C_{9}$ is even favoured but much harder to explain in the context of existing models. We refer to [102] for tables with various solutions.

Previous discussions of this actual topic can be found in [93, 103-110]. It should be emphasized that these analyses are subject to theoretical uncertainties, which have been discussed at length in $[103,111-118]$ and it remains to be seen whether the observed anomalies are only result of statistical fluctuations and/or underestimated error uncertainties.

As far as theoretical uncertainties are concerned much cleaner is the ratio

$$
\mathscr{R}_{K}^{\mu e}=\frac{\mathscr{B}\left(B^{+} \rightarrow K^{+} \mu^{+} \mu^{-}\right)}{\mathscr{B}\left(B^{+} \rightarrow K^{+} e^{+} e^{-}\right)}=0.745_{-0.074}^{+0.090}(\text { stat }) \pm 0.036(\text { syst })
$$

where the quoted value is the one from LHCb. It is by $2.6 \sigma$ lower than its SM value: $1+\mathscr{O}\left(10^{-4}\right)$ and is an intriguing signal of the breakdown of lepton flavour universality.

There is some consensus in the community that all these anomalies can be most naturally reproduced with the help of tree-level $Z^{\prime}$ contribution with $Z^{\prime}$ having only left-handed flavour violating couplings to quarks $[34,93,100,101,119-122]{ }^{5}$ or leptoquark exchanges $[33,34,125-128]$, even if there exist other explanations. As $C_{9}$ and $C_{10}$ involve vector and axial vector couplings to muons, respectively, the $S U(2)$ relation in (6.1) implies then the values of neutrino couplings. As the $Z^{\prime}$ couplings to quarks entering the ratios $R_{i}$ listed above are the same, the present anomalies imply then uniquely [34]

$$
\mathscr{R}_{K}>1, \quad \mathscr{R}_{K^{*}}>1 \quad\left(Z^{\prime}\right) .
$$

A similar exercise in the case of SM $Z$ boson, where the leptonic couplings are known, implies [34]

$$
\mathscr{R}_{K}<1, \quad \mathscr{R}_{K^{*}}<1 \quad(Z) .
$$

In this manner one could distinguish between these two scenarios.

It is instructive to see how this different behaviour arises. In the presence of the dominance of left-handed flavour violating quark currents, both in the SM and beyond it, only one operator with $(V-A) \otimes(V-A)$ structure contributes and its Wilson coefficient is

$$
C_{L}=C_{L}^{\mathrm{SM}}+C_{L}^{\mathrm{NP}}, \quad C_{L}^{\mathrm{SM}}<0 .
$$

Now the products of $Z^{\prime}$ left-handed flavour violating quark couplings and of the muon couplings $\Delta_{V}^{\mu \bar{\mu}}\left(Z^{\prime}\right)$ and $\Delta_{A}^{\mu \bar{\mu}}\left(Z^{\prime}\right)$ build the Wilson coefficients $C_{9}^{\mathrm{NP}}$ and $C_{10}^{\mathrm{NP}}$, respectively. The invariance of NP under the SM gauge group implies then, as seen in (6.1),

$$
C_{L}^{\mathrm{NP}}=\frac{C_{9}^{\mathrm{NP}}-C_{10}^{\mathrm{NP}}}{2} \approx C_{9}^{\mathrm{NP}},
$$

with the second relation following from the $b \rightarrow s \mu^{+} \mu^{-}$data (7.5). On the other hand in the $Z$ case one has

$$
C_{L}^{\mathrm{NP}}=C_{10}^{\mathrm{NP}} \approx-C_{9}^{\mathrm{NP}}, \quad(Z) .
$$

\footnotetext{
${ }^{5}$ The $Z^{\prime}$ in 331 models has lepton flavour universal couplings [97,98,123,124] but can still solve the $B \rightarrow K^{*} \mu^{+} \mu^{-}$ anomaly and improve on $B_{s} \rightarrow \mu^{+} \mu^{-}$. See below.
} 
With $C_{L}^{\mathrm{SM}}<0$ and $C_{9}^{\mathrm{NP}}<0$ one obtains automatically the results in (7.7) and (7.8). If $C_{10}^{\mathrm{NP}}=0$ and $C_{9}^{\mathrm{NP}}<0$, then $Z$ does not contribute but again (7.7) is valid.

Yet from my point of view a closer look indicates that a $Z^{\prime}$ is the favourite scenario in this case for two reasons. First, the vector coupling of $Z$ to muons, relevant for $C_{9}$, is small. Second, $\mathscr{R}_{K}^{\mu e} \neq 1$ being a signal of violation of lepton universality can easily be arranged in the case of a $Z^{\prime}$. This is basically impossible in the case of $Z$.

Now, generally the presence of $Z^{\prime}$ with flavour violating couplings generates through $Z-Z^{\prime}$ mixing such couplings for $Z$ so that both $Z^{\prime}$ and $Z$ contribute. In this case one finds in place of (7.10)

$$
C_{L}^{\mathrm{NP}}=\frac{C_{9}^{\mathrm{NP}}-C_{10}^{\mathrm{NP}}}{2}+3 \frac{C_{Z}}{2}, \quad\left(Z^{\prime}, Z\right),
$$

where $C_{Z}$ depends on $Z-Z^{\prime}$ mixing. As this mixing is model dependent nothing concrete can be concluded without having a specific model. This shows the superiority of the top-down approach over the bottom-up approach. The case of 331 models, which we will summarize now, illustrates this in a transparent manner.

\subsection{Models}

A concrete example for $Z^{\prime}$ tree-level FCNC is a model based on the gauge group $S U(3)_{C} \times$ $S U(3)_{L} \times U(1)_{X}$, the so-called 331 model, originally developed in $[129,130]$. There are different versions of the 331 model characterized by a parameter $\beta$ that determines the particle content. The value of $\beta$ specifies also the leptonic couplings of $Z^{\prime}$ so that this model is much more predictive than general $Z^{\prime}$ models. In three detailed papers [96-98] we have analyzed various aspects of flavour violation in these models finding numerous correlations between various observables ${ }^{6}$. I will summarize here only the last of these analyzes as this paper is more complete than the previous two. It includes the effects of $Z-Z^{\prime}$ mixing which were neglected previously in the literature and also in our two first papers. This turns out to be justified for $\Delta F=2$ processes and $B \rightarrow$ $K^{*} \mu^{+} \mu^{-}$but for certain values of $\beta$ the contribution from the induced FCNCs mediated by $Z$ cannot be neglected. A new aspect of this paper is the correlation of flavour violating effects with the electroweak precision observables which allows to select the favourite values of $\beta$ and further selection will be possible when the flavour data improve. Moreover, [98] investigates the dependence on the fermion representations, presenting the results for two cases. After constraints from $\Delta F=2$ transitions have been imposed, in addition to $\beta$ and $M_{Z^{\prime}}$, the only new parameter is $\tan \bar{\beta}$ that together with $\beta$ describes the $Z-Z^{\prime}$ mixing.

This analysis shows very clearly the superiority of the top-down approach over the bottomup approach. The correlations between various flavour observables as functions of the size of $Z-Z^{\prime}$ mixing and the correlations with electroweak observables are totally beyond the bottom-up approach and such correlations will be vital in the flavour precision era. The interested reader is invited to look at numerous plots in [98] so that she (he) can better appreciate the statements just made. In what follows I will just summarize the most interesting results of this study.

As far as flavour physics is concerned our main findings are as follows:

\footnotetext{
${ }^{6}$ There are other analyses of flavour physics which are referred to in [98] and in [131].
} 
- NP contributions to $\Delta F=2$ transitions and decays like $B \rightarrow K^{*} \ell^{+} \ell^{-}$are governed by $Z^{\prime}$ tree-level exchanges.

- On the other hand for $B_{s, d} \rightarrow \mu^{+} \mu^{-}$decays $Z$ contributions can be important. We find that for $\tan \bar{\beta}=5.0$ these contributions interfere constructively with $Z^{\prime}$ contributions enhancing NP effects, while for low $\tan \bar{\beta}=0.2 Z$ contributions practically cancel the ones from $Z^{\prime}$.

- Similarly $Z$ boson tree-level contributions to $B_{s, d}$ and $K$ decays with neutrinos in the final state can be relevant but in this case the $\tan \bar{\beta}$ dependence is opposite to the one found for $B_{s, d} \rightarrow \mu^{+} \mu^{-}$. We find that for $\tan \bar{\beta}=5.0$ these contributions practically cancel the ones from $Z^{\prime}$ but for low $\tan \bar{\beta}=0.2 Z$ contributions interfere constructively with $Z^{\prime}$ contributions enhancing NP effects.

- As a result of this opposite dependence on $\tan \bar{\beta}$ the correlations between decays with muons and neutrinos in the final state exhibit significant dependence on $\tan \bar{\beta}$ and can serve to determine this parameter in the future.

- Our analysis of $\varepsilon^{\prime} / \varepsilon$ is to our knowledge the first one in 331 models. Including both $Z^{\prime}$ and $Z$ contributions we find that the former dominate but NP effects are not large.

- We also find a strict correlation between $\varepsilon^{\prime} / \varepsilon$ and $\mathscr{B}\left(K_{L} \rightarrow \pi^{0} v \bar{v}\right)$. The interesting feature here, is the decrease of $\varepsilon^{\prime} / \varepsilon$ with increasing $\mathscr{B}\left(K_{L} \rightarrow \pi^{0} v \bar{v}\right)$ for negative $\beta$ and its increase with increasing $\mathscr{B}\left(K_{L} \rightarrow \pi^{0} v \bar{v}\right)$ for positive $\beta$.

- Imposing the electroweak precision constraints only seven among 24 combinations of $\beta$, $\tan \bar{\beta}$ and two fermion representation $F_{1}$ or $F_{2}$ provide better or equally good description of the electroweak precision data compared with the SM. Interestingly, among these models none of them allows to simultaneously suppress the rate for $B_{s} \rightarrow \mu^{+} \mu^{-}$and soften the $B_{d} \rightarrow K^{*} \mu^{+} \mu^{-}$anomaly. But there are few models which either suppress the rate for $B_{s} \rightarrow$ $\mu^{+} \mu^{-}$or soften the $B_{d} \rightarrow K^{*} \mu^{+} \mu^{-}$anomaly. Yet, none of these models allows significant NP effects in $B$ and $K$ decays with neutrinos in the final state although departures by $15 \%$ relative to the SM prediction for the rate of $K_{L} \rightarrow \pi^{0} v \bar{v}$ are still possible.

\section{The Power of $B \rightarrow K^{(*)} v \bar{v}, K^{+} \rightarrow \pi^{+} v \bar{v}$ and $K_{L} \rightarrow \pi^{0} v \bar{v}$ Decays}

8.1 $B \rightarrow K^{(*)} v \bar{v}$

We have just seen that these decays, when measured, could allow to distinguish between various explanations of the present anomalies in $b \rightarrow s \mu^{+} \mu^{-}$transitions. But it should be stressed that these decays are of interest on its own as they are theoretically cleaner than $B \rightarrow K^{(*)} \mu^{+} \mu^{-}$and allow good tests of the presence of right-handed currents and in general of NP.

Both decays should be measured at Belle II. The most recent estimate of their branching ratios within the SM [34] reads:

$$
\mathscr{B}\left(B^{+} \rightarrow K^{+} v \bar{v}\right)=\left[\frac{\left|V_{c b}\right|}{0.0409}\right]^{2}(4.0 \pm 0.4) \times 10^{-6},
$$




$$
\mathscr{B}\left(B^{0} \rightarrow K^{* 0} v \bar{v}\right)=\left[\frac{\left|V_{c b}\right|}{0.0409}\right]^{2}(9.2 \pm 0.9) \times 10^{-6},
$$

where the errors in the parentheses are fully dominated by form factor uncertainties. We expect that when these two branching ratios will be measured, these uncertainties will be further decreased and $\left|V_{c b}\right|$ will be precisely known so that a very good test of the SM will be possible.

But in the context of such tests one should still take care of non-perturbative tree level contributions from $B^{+} \rightarrow \tau^{+} v$ to $B^{+} \rightarrow K^{+} v \bar{v}$ and $B^{+} \rightarrow K^{*+} v \bar{v}$ at the level of roughly (5-10)\% which have been pointed out [132]. This should be possible in the future ones the data on $B^{+} \rightarrow \tau^{+} v$ will be precise. The SM results quoted above refer only to the short-distance contributions.

An extensive analysis of these decays model-independently and in various extensions of the SM has been performed in [34]. In addition to the correlations between various ratios $R_{i}$ discussed for $Z^{\prime}$ models, shown in figure 5 of that paper, of particular interest are the correlations between $\mathscr{R}_{K}$ and $\mathscr{R}_{K^{*}}$ in various scalar and vector leptoquarks models with leptoquarks carrying different quantum numbers. Figure 10 in [34] shows that precise measurements of $\mathscr{R}_{K}$ and $\mathscr{R}_{K^{*}}$ could distinguish between various leptoquark models.

Also figures 1, 2, 3 and 11 in [34] demonstrate very clearly that we will have a lot of fun when the experimental data on $B \rightarrow K^{(*)} \mu^{+} \mu^{-}$and $B \rightarrow K^{(*)} v \bar{v}$ will be known and the theoretical issues in $B \rightarrow K^{(*)} \mu^{+} \mu^{-}$are clarified. But we will have even more fun when the branching ratios on $K^{+} \rightarrow \pi^{+} v \bar{v}$ and $K_{L} \rightarrow \pi^{0} v \bar{v}$ will be measured.

\section{2 $K^{+} \rightarrow \pi^{+} v \bar{v}$ and $K_{L} \rightarrow \pi^{0} v \bar{v}$ in the SM}

In view of the recent start of the NA62 experiment at CERN which is expected to measure the $K^{+} \rightarrow \pi^{+} v \bar{v}$ branching ratio with the precision of $10 \%$ [133,134], we have recently summarized the present status of this decay within the SM and of $K_{L} \rightarrow \pi^{0} v \bar{v}$ which should be measured by KOTO experiment around 2020 at J-PARC [135, 136]. As the perturbative QCD [137-142] and electroweak corrections [143-145] in both decays are fully under control, the present uncertainties in the branching ratios originate within the SM dominantly from $\left|V_{c b}\right|,\left|V_{u b}\right|$ and $\gamma$ when extracted from tree-level decays. Unfortunately the clarification of the discrepancies between inclusive and exclusive determinations of $\left|V_{c b}\right|$ and $\left|V_{u b}\right|$ from tree-level decays are likely to be resolved only at the time of the Belle II experiment at SuperKEKB at the end of this decade. Therefore, we investigated whether in the coming years higher precision on both branching ratios can be obtained by eliminating $\left|V_{c b}\right|,\left|V_{u b}\right|$ and $\gamma$ with the help of other observables that are already precisely measured. In this context $\varepsilon_{K}$ and $\Delta M_{s, d}$, accompanied by recent and future progress in QCD lattice calculations, as well as the improved measurements of mixing induced CP asymmetries $S_{\psi K_{S}}$ and $S_{\psi \phi}$ at the LHC will play prominent roles.

We find [23]

$$
\mathscr{B}\left(K^{+} \rightarrow \pi^{+} v \bar{v}\right)=(9.1 \pm 0.7) \times 10^{-11}, \quad \mathscr{B}\left(K_{L} \rightarrow \pi^{0} v \bar{v}\right)=(3.0 \pm 0.3) \times 10^{-11}
$$

which is roughly by a factor of two more precise than using present tree-level values of $\left|V_{c b}\right|,\left|V_{u b}\right|$ and $\gamma$ in (2.4) and (2.5).

We also find

$$
\left|V_{c b}\right|=(42.4 \pm 1.2) \times 10^{-3}, \quad\left|V_{u b}\right|=(3.61 \pm 0.14) \times 10^{-3}, \quad \gamma=(69.5 \pm 5.0)^{\circ}
$$



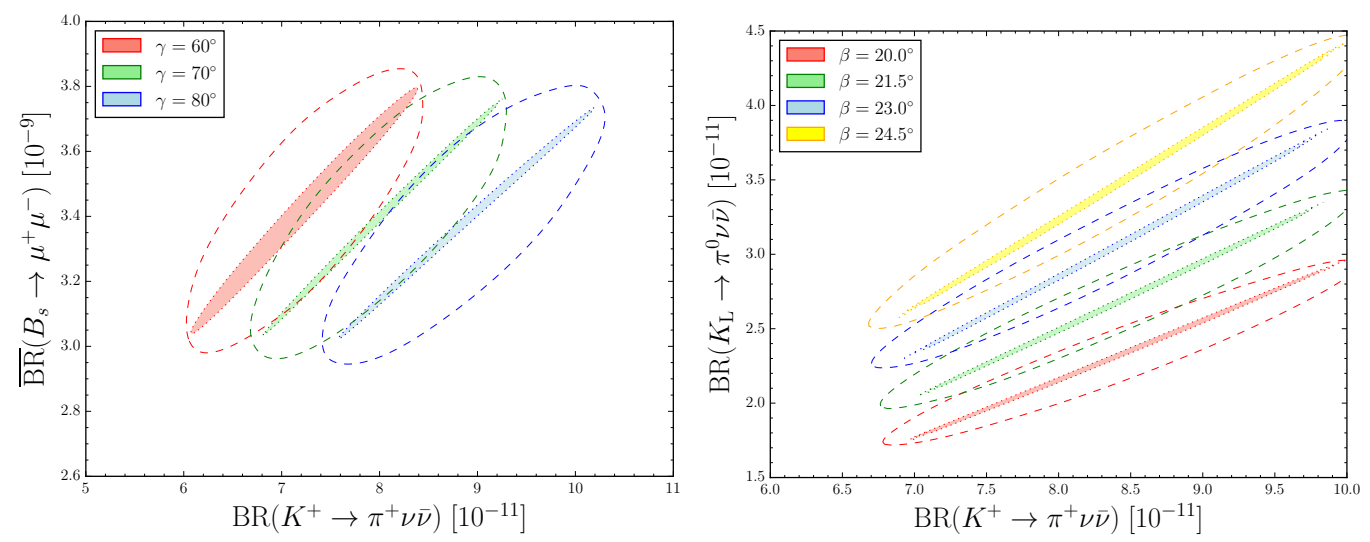

Figure 7: Left panel: correlation of $\overline{\mathscr{B}}\left(B_{s} \rightarrow \mu^{+} \mu^{-}\right)$versus $\mathscr{B}\left(K^{+} \rightarrow \pi^{+} v \bar{v}\right)$ for fixed values of $\gamma$. Right panel: correlation of $\mathscr{B}\left(K_{L} \rightarrow \pi^{0} v \bar{v}\right)$ versus $\mathscr{B}\left(K^{+} \rightarrow \pi^{+} v \bar{v}\right)$ for fixed values of $\beta$. In both plots the dashed regions correspond to a $68 \%$ CL resulting from the uncertainties on all other inputs, while the inner filled regions result from including only the uncertainties from the remaining CKM inputs.

in this manner. The large value of $\left|V_{c b}\right|$ is fully consistent with its inclusive determinations and is required by the data on $\varepsilon_{K}$. We note that with the reduced error on the $\Delta B=2$ paramete $\xi$, promised in [146], the error on $\gamma$ will decrease down to $2.3^{\circ}$.

The indirect fits done by UTfit [147] and CKMfitter [148] that are summarized in [15] yield

$$
\begin{aligned}
& \text { UTfit: } \quad\left|V_{u b}\right|=(3.63 \pm 0.12) \times 10^{-3}, \quad\left|V_{c b}\right|=(41.7 \pm 0.6) \times 10^{-3} \text {, } \\
& \text { CKMfitter: } \quad\left|V_{u b}\right|=\left(3.57_{-0.31}^{+0.41}\right) \times 10^{-3}, \quad\left|V_{c b}\right|=\left(41.4_{-1.8}^{+1.4}\right) \times 10^{-3}
\end{aligned}
$$

in very good agreement with our results. We note however, that these two groups included in their analyses the information from tree level decays, whereas we decided not to use it because of discrepancies between inclusive and exclusive determinations of $\left|V_{u b}\right|$ and $\left|V_{c b}\right|$. We also left out tree level determination of $\gamma$ from this fit.

In my view one of the highlights of [23] is the new correlation between $\mathscr{B}\left(K^{+} \rightarrow \pi^{+} v \bar{v}\right)$, $\mathscr{B}\left(B_{s} \rightarrow \mu^{+} \mu^{-}\right)$and $\gamma$ extracted from tree-level decays within the SM that is only very weakly dependent on other CKM parameters, in particular $\left|V_{u b}\right|$ and $\left|V_{c b}\right|$. This correlation should be of interest to CERN experimentalists, that is LHCb, CMS and NA62, who in the coming years will significantly improve the measurements on these three quantities. We show it in the left panel of Fig. 7. We observe that the central experimental value of $\overline{\mathscr{B}}\left(B_{S} \rightarrow \mu^{+} \mu^{-}\right)$, that is below $3.0 \times 10^{-9}$, implies $\mathscr{B}\left(K^{+} \rightarrow \pi^{+} v \bar{v}\right)$ in the ballpark $7.0 \times 10^{-11}$. If confirmed, this would possibly make the identification of NP in latter decay easier.

There are other interesting correlations presented in [23], like the update of the correlation between branching ratios for $K^{+} \rightarrow \pi^{+} v \bar{v}$ and $K_{L} \rightarrow \pi^{0} v \bar{v}$ and $S_{\psi K_{S}}$ that is practically independent of $\left|V_{u b}\right|$ and $\left|V_{c b}\right|[43,44]$. We show this correlation in the right panel of Fig. 7. Moreover, we provide a number of rather accurate expressions that should allow one to monitor easily the future experimental and theoretical progress on the observables in question.

\section{$8.3 K^{+} \rightarrow \pi^{+} v \bar{v}$ and $K_{L} \rightarrow \pi^{0} v \bar{v}$ beyond the SM}

Beyond the SM both branching ratios can still be significantly enhanced but the size of this 
enhancement strongly depends on NP scenario considered and the constraints in a given scenario coming from other observables. A new analysis in progress and other analyses by us, in particular in [149], allow us to draw a general picture of NP contributions to the two decays in question:

- In models with MFV and $U(2)^{3}$ models NP effects amount to at most $20-30 \%$ making the distinction from the SM difficult. The charcteristic feature in these scenarios is the strong correlation between both branching ratios with both either enhanced or suppressed relative to the SM.

- In non-MFV models the shape of the correlation depends on whether $\varepsilon_{K}$ constraint is important or not [150]. If it is, the shape of this correlation is as shown in the left-panel of Fig. 8. The upper brunch is characteristic for MFV, while the lower one shows a non-MFV behaviour: the branching ratio for $K^{+} \rightarrow \pi^{+} v \bar{v}$ is modified while the one for $K_{L} \rightarrow \pi^{0} v \bar{v}$ remains basically unchanged. On the other hand if with sufficient number of parameters $\varepsilon_{K}$ constraint can be eliminated then the correlation has the shape shown in the right panel of Fig. 8 .

- The size of possible enhancements of $\mathscr{B}\left(K_{L} \rightarrow \pi^{0} v \bar{v}\right)$ is bounded by $\varepsilon^{\prime} / \varepsilon$. The size of possible enhancements of $\mathscr{B}\left(K^{+} \rightarrow \pi^{+} v \bar{v}\right)$ by $K_{L} \rightarrow \mu^{+} \mu^{-}$and $\varepsilon^{\prime} / \varepsilon$. This has been known already for many years [1] but in view of the improved determination of electroweak contribution to $\varepsilon^{\prime} / \varepsilon$ with the help of lattice QCD, such studies become more quantitative. We refer to [149] for details.

- In a scenario with arbitrary flavour-violating $Z$ couplings to quarks the correlation of $K_{L} \rightarrow$ $\pi^{0} v \bar{v}$ with $\varepsilon^{\prime} / \varepsilon$ very significantly limits possible enhancements of $\mathscr{B}\left(K_{L} \rightarrow \pi^{0} v \bar{v}\right)$ but enhancements by a factor $3-4$ are still possible. $\mathscr{B}\left(K^{+} \rightarrow \pi^{+} v \bar{v}\right)$ can be in particular enhanced when $K_{L} \rightarrow \mu^{-} \mu^{+}$can be eliminated. This is the case of LH and RH flavour violating $Z$ couplings being approximately equal. Also when only $\mathrm{RH}$ couplings are present. Therefore values of $\mathscr{B}\left(K^{+} \rightarrow \pi^{+} v \bar{v}\right)$ in the ballpark of $(15-25) 10^{-11}$ are still possible.

- Larger enhancements are still possible in the $Z^{\prime}$ scenarios with arbitrary flavour-violating couplings. Here, in contrast to $Z$ models the diagonal quark couplings, required for $\varepsilon^{\prime} / \varepsilon$ are only known in concrete models. While in the 331 model, discussed previously, $Z^{\prime}$ effects have been found to be small [98], in general $Z^{\prime}$ scenarios a strict correlation between $\varepsilon^{\prime} / \varepsilon$ and both rare decays does not really exist and larger effects are still be allowed.

\section{Can We Reach the Zeptouniverse with Rare $K$ and $B_{s, d}$ Decays?}

We will finally address the central issue of our paper, the reach of flavour physics in testing very short distances scales. This issue becomes relevant independently of wheather LHC will discover new particles or not. The point is that LHC will directly probe only distance scales down to $10^{-19} \mathrm{~m}$, corresponding to energy scales at the level of a few $\mathrm{TeV}$. This is of course impressive but one would like to know what happens at even shorter distance scales. In order to reach even higher resolution before the advent of future high-energy colliders, it is necessary to consider indirect 
probes of NP, a prime example being $\Delta F=2$ neutral meson mixing processes, which are sensitive to much shorter distance scales.

In fact in the framework of effective theories, the analyses in [27-29], which dealt dominantly with $\Delta F=2$ observables, have shown that in the presence of left-right operators and $\mathscr{O}(1)$ couplings one could be in principle sensitive to scales as high as $10^{4} \mathrm{TeV}$, or even higher energy scales. Unfortunately, as pointed out in [151], $\Delta F=2$ observables alone will not really give us significant information about the particular nature of this NP. This is related to the symmetric structure of the formulae like the one in (5.3): one cannot distinguish between left and right by using only $\Delta F=2$ transitions. On the other hand our DNA charts demonstrate that $\Delta F=1$ processes, in particular rare $K$ and $B_{s, d}$ decays, can help us in this matter through various correlations between observables that depend on whether left-handed or right-handed couplings are involved.

But as left-right operators involving four quarks are not the driving force in these decays, which generally contain operators built out of one quark current and one lepton current, it is not evident that rare $K$ and $B_{s, d}$ decays can help us in reaching the Zeptouniverse even in the flavour precision era. This interesting question has been addressed in [151] and I would like to summarize the results of this study.

Certainly the answer to this question depends on the size of NP, its nature and in particular on the available precision of experiments and of the SM predictions for flavour observables. The latter precision depends on the extraction of CKM parameters from the data and on the theoretical uncertainties. Both are expected to be reduced in this decade down to $1-2 \%$, which should allow NP to be identified even if it contributed only at the level of $20-30 \%$ to the branching ratios.

In order to find the maximal resolution one has to decide what is the largest coupling of NP particles to SM particles still consistent with perturbativity. A coupling of at most 3.0 at the high scale seems to be a reasonable choice. The results for resolutions quoted below depend on this number linearly. For smaller couplings the resolutions are worse.

Answering this question first in the context of $Z^{\prime}$ tree-level exchanges our main findings are as follows:

- Future precise measurements of several $\Delta F=1$ observables and in particular correlations between them can distinguish between LH and RH currents, but the maximal resolution consistent with perturbativity strongly depends on whether only LH or only RH or both LH and $\mathrm{RH}$ flavour changing $Z^{\prime}$ couplings to quarks are present in nature.

- If $Z^{\prime}$ has only LH or RH couplings we can in principle reach scales of $200 \mathrm{TeV}$ and $15 \mathrm{TeV}$ for $K$ and $B_{s, d}$, respectively. These numbers depend on the room left for NP in $\Delta F=2$ observables, which have an important impact on the resolution available in these NP scenarios. In the left panel in Fig. 8 we show the result of $K \rightarrow \pi v \bar{v}$ for $M_{Z^{\prime}}=50 \mathrm{TeV}$.

- Smaller distance scales can only be resolved if both RH and LH couplings are present in order to cancel the NP effects in the $\Delta F=2$ observables. Simply having more free parameters one can easier satisfy $\Delta F=2$ constraints without relevant impact on $\Delta F=1$ transistions. But to achieve this some tuning of couplings is required. In particular RH and LH couplings have to differ considerably from each other. This large hierarchy of couplings is dictated primarily by the ratio of hadronic matrix elements of $\mathrm{LR} \Delta F=2$ operators and those for LL and RR 
operators (see comments after (5.3)) and by the room left for NP in $\Delta F=2$ processes. Future advances in the determination of CKM parameters and calculation of the relevant hadronic matrix elements should specify this room precisely. We find that in this case the scales as high as $2000 \mathrm{TeV}$ can be reached with the help of $K^{+} \rightarrow \pi^{+} v \bar{v}$ and $K_{L} \rightarrow \pi^{0} v \bar{v}$ and $160 \mathrm{TeV}$ with the help $B_{s, d} \rightarrow \mu^{+} \mu$ when $Z^{\prime}$ is at work. In the right panel in Fig. 8 we show the result of $K \rightarrow \pi v \bar{v}$ for $M_{Z^{\prime}}=500 \mathrm{TeV}$. We observe that the structure of this correlation is very different from the case when $\Delta F=2$ constraint is present. We discuss this issue below.

- A study of tree-level (pseudo-)scalar exchanges shows that in this case $B_{s, d} \rightarrow \mu^{+} \mu^{-}$can probe scales up to $750 \mathrm{TeV}$, both for scenarios with purely $\mathrm{LH}$ or RH scalar couplings to quarks and for scenarios allowing for both LH and RH couplings. For the limit of a degenerate scalar and pseudoscalar NP effects in $\Delta F=2$ observables can cancel even without imposing a tuning on the couplings. The outcome of this analysis is shown in Fig. 9.

- We have discussed models with several gauge bosons. Also in this case the basic strategy for being able to explore very high energy scales is to break the stringent correlation between $\Delta F=1$ and $\Delta F=2$ processes and to suppress NP contributions to the latter without suppressing NP contributions to rare decays. The presence of a second heavy neutral gauge boson allows us to achieve the goal with only LH or RH currents by applying an appropriate tuning.

- While the highest achievable resolution in the presence of several gauge bosons is comparable to the case of a single gauge boson because of the perturbativity bound, the correlations between $\Delta F=1$ observables could differ from the ones presented here. This would be in particular the case if LH and RH couplings of these bosons where of similar size. But a detailed study of such scenarios would require the formulation of concrete models.

- If FCNCs only occur at one loop level the highest energy scales that can be resolved for maximal couplings are typically reduced relative to the case of tree-level FCNCs by a factor of at least 3 and 6 for $\Delta F=1$ and $\Delta F=2$ processes, respectively.

Now comes the following difficulty, which requires further study. The observables depend generally on the ratio of the coupling and the mass of the exchanged object. Therefore if in the future one will see some deviations from the SM, to first approximation, only this ratio will be determined and at first side without knowing the coupling we will not know which scales are involved. However, the case of $K^{+} \rightarrow \pi^{+} v \bar{v}$ and $K_{L} \rightarrow \pi^{0} v \bar{v}$ decays, as seen in Fig. 8, allows to get an idea which scales are involved and this is because one of the decays $\left(K_{L} \rightarrow \pi^{0} v \bar{v}\right)$ is $\mathrm{CP}$-violating. If $\varepsilon_{K}$ constraint is vital then the structure of correlation is as seen in the left panel of this figure. But when it is eliminated, the new phase in the decays is free and a different correlation is seen in the right panel allowing the two branching ratios to take values which are not allowed if $\Delta F=2$ constraint is relevant. The analytic understanding of this difference has been provided in [150]. Thus if in the future such values will be measured this indeed could be a signal that we deal here with very high scales. Moreover, this would indicate that both left-handed and right-handed currents in $\Delta F=2$ processes at work, thereby allowing $\Delta F=2$ constraints to be satisfied. 

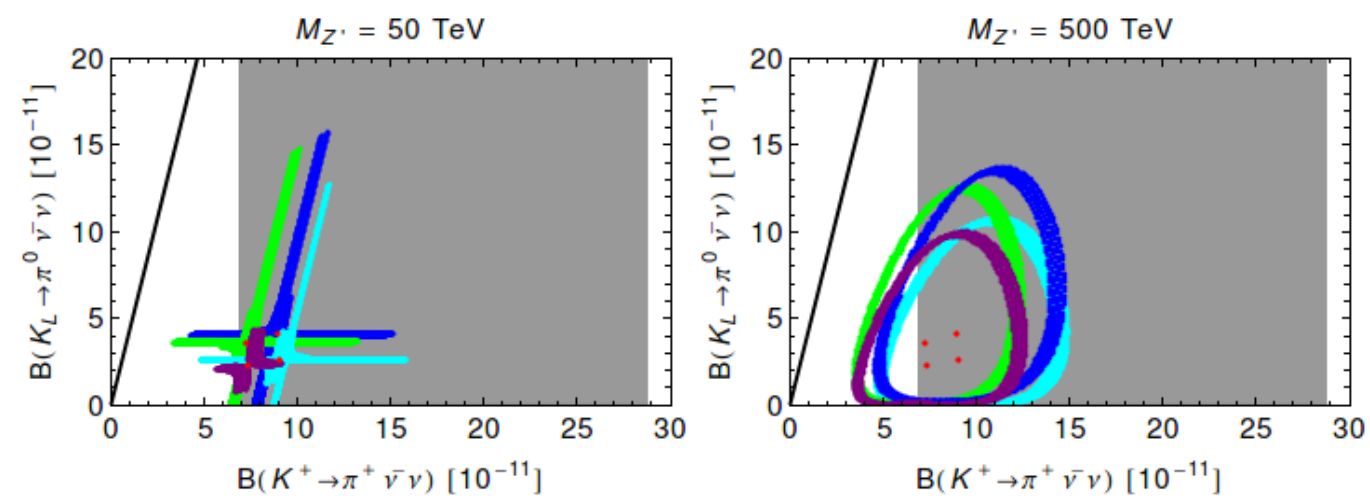

Figure 8: $\mathscr{B}\left(K_{L} \rightarrow \pi^{0} v \bar{v}\right)$ versus $\mathscr{B}\left(K^{+} \rightarrow \pi^{+} v \bar{v}\right)$ for $M_{Z^{\prime}}=50 \mathrm{TeV}$ in the LHS (left) and for $M_{Z^{\prime}}=$ $500 \mathrm{TeV}$ in $L+R$ scenario. The colours distinguish between different CKM input which also implies the four red points corresponding to the SM central values for these four CKM scenarios, respectively. The black line corresponds to the Grossman-Nir bound [152]. The gray region shows the experimental range of $\left.\mathscr{B}\left(K^{+} \rightarrow \pi^{+} v \bar{v}\right)\right)_{\text {exp }}=\left(17.3_{-10.5}^{+11.5}\right) \times 10^{-11}$. From [151].

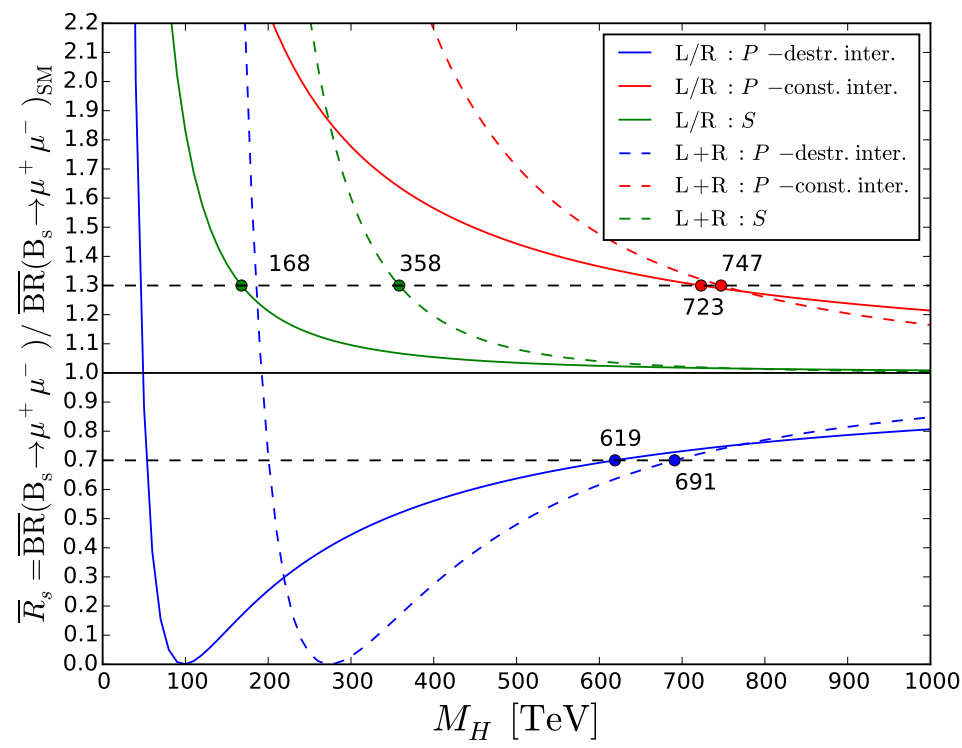

Figure 9: Dependence of $\bar{R}_{s}$ on the heavy scalar mass $M_{H}$, showing the pure $L H($ or $R H)$ scenario and the combined L+R scenario (see [151] for details).

But when two $\mathrm{CP}$ conserving quantities are involved, the correlations between observables in $B_{s, d}$ decays turn out to have similar shape independently whether both left-handed and right-handed couplings are at work. Therefore in this case it is more difficult to find out which energy scales are involved. Still we have presented a simple idea for a rough indirect determination of $M_{Z^{\prime}}$ by means of the next linear $e^{+} e^{-}$or $\mu^{+} \mu^{-}$collider and precision flavour data. It uses the fact that the LR operators present in $\Delta F=2$ transitions have large anomalous dimensions so that $M_{Z^{\prime}}$ can be determined through renormalisation group effects provided it is well above the LHC scales.

In summary we have demonstrated in [151] that NP with a particular pattern of dynamics could be investigated through rare $K$ and $B_{s, d}$ decays even if the scale of this NP would belong to the Zeptouniverse. As expected from other studies it is in principle easier to reach the Zeptouniverse 
with the help of rare $K$ decays than $B_{s, d}$ decays. However, this assumes the same maximal couplings in these three systems and this could be not the case. Moreover, in the presence of tree-level pseudoscalar exchanges very short distance scales can be probed by $B_{s, d} \rightarrow \mu^{+} \mu^{-}$decays.

We should emphasise that although the main goal in [151] was to reach the highest energy scales with the help of rare decays, it will of course be exciting to explore any scale of NP above the LHC scales in this decade. Moreover, we still hope that high energy proton-proton collisions at the LHC will exhibit at least some footprints of new particles and forces. This would greatly facilitate flavour analyses as the one just presented.

Finally, it should not be forgotten that in principle much higher energy scales or much better resolution could be achieved in the future with the help of charged lepton flavour violating decays such as $\mu \rightarrow e \gamma, \mu \rightarrow 3 e$ and $\tau \rightarrow 3 \mu, \mu \rightarrow e$ conversion in nuclei, and electric dipole moments $[62,64,153-160]$. But this topic is another story.

\section{Final Remarks}

It is clear that presently available precision on the multitude of observables considered in our papers and briefly in this lecture is insufficient for the execution of the flavour program outlined by us. Yet the coming flavour precision era, in which the measurements of most observables listed in Fig. 4 will reach much higher precision and lattice QCD calculations will be significantly improved, should allow us to obtain at least a rough picture of the physics beyond the LHC and if we are lucky even reach the Zeptouniverse. This would be a very important step towards the construction of a fundamental theory of particles and interactions.

Having this in mind I do not share the frustration of some of my colleagues caused by the lack of NP signals in high energy collisions at the LHC or difficulties in finding dark matter particles and axions. One should not forget that low energy processes and in particular rare processes like rare kaon decays and CP violation were vital in the construction of the SM well before the discovery of $W$ and $Z$ bosons, of the top quark and the Higgs. While I hope very much that in the coming years LHC will discover new particles, this lecture shows that particle physics will still have much to offer if this will turn out not to be the case.

\section{Acknowledgements}

I thank all my collaborators, in particular Dario Buttazzo, Fulvia De Fazio, Jennifer GirrbachNoe and Rob Knegjens, Christoph Niehoff and David Straub for exciting time we spent together exploring recently the short distance scales with the help of flavour violating processes. Finally I would like to thank the organizers of FWNP for their hospitality and perfect organization of this memorable event. The research presented in this report was dominantly financed and done in the context of the ERC Advanced Grant project "FLAVOUR" (267104). It was also partially supported by the DFG cluster of excellence "Origin and Structure of the Universe".

\section{References}

[1] A. J. Buras and J. Girrbach, Towards the Identification of New Physics through Quark Flavour Violating Processes, Rept.Prog.Phys. 77 (2014) 086201, [arXiv: 1306.3775$].$

[2] A. J. Buras, Towards the Identification of New Physics through Correlations between Flavour Observables, PoS EPS-HEP2013 (2013) 331, [arXiv: 1309 . 7791]. 
[3] E. Lunghi and A. Soni, Possible Indications of New Physics in $B_{d}$-mixing and in $\sin (2 \beta)$ Determinations, Phys. Lett. B666 (2008) 162-165, [arXiv: 0803.4340$].$

[4] A. J. Buras and D. Guadagnoli, Correlations among new CP violating effects in $\Delta F=2$ observables, Phys. Rev. D78 (2008) 033005, [arXiv: 0805 . 3887].

[5] SWME Collaboration, J. A. Bailey, Y.-C. Jang, W. Lee, and S. Park, Standard Model evaluation of $\varepsilon_{K}$ using lattice $Q C D$ inputs for $\hat{B}_{K}$ and $V_{c b}$, arXiv: 1503.05388.

[6] R. Fleischer, Towards New Frontiers in CP Violation in B Decays, J.Phys.Conf.Ser. 556 (2014), no. 1 012035, [arXiv:1408.2497].

[7] C. Bobeth, U. Haisch, A. Lenz, B. Pecjak, and G. Tetlalmatzi-Xolocotzi, On new physics in $\Delta \Gamma_{d}$, JHEP 1406 (2014) 040, [arXiv: 1404.2531$].$

[8] C. Bobeth, M. Gorbahn, and S. Vickers, Weak annihilation and new physics in charmless $B \rightarrow M M$ decays, arXiv:1409.3252.

[9] J. Brod, A. Lenz, G. Tetlalmatzi-Xolocotzi, and M. Wiebusch, New physics effects in tree-level decays, arXiv:1412.1446.

[10] A. X. El-Khadra, Quark Flavor Physics Review, PoS LATTICE2013 (2014) 001, [arXiv:1403.5252].

[11] M. Beneke, G. Buchalla, M. Neubert, and C. T. Sachrajda, $Q C D$ factorization for $B \rightarrow K \pi, \pi \pi$ decays: Strong phases and CP violation in the heavy quark limit, Phys.Rev.Lett. 83 (1999) 1914-1917, [hep-ph/9905312].

[12] A. J. Buras, Climbing NLO and NNLO Summits of Weak Decays, arXiv:1102 . 5650.

[13] A. J. Buras and J. Girrbach, Complete NLO QCD Corrections for Tree Level Delta F $=2$ FCNC Processes, JHEP 1203 (2012) 052, [arXiv: 1201.1302 ].

[14] A. J. Buras and J. Girrbach, Completing NLO QCD Corrections for Tree Level Non-Leptonic Delta F = 1 Decays Beyond the Standard Model, JHEP 1202 (2012) 143, [arXiv: 1201.2563 ].

[15] G. Ricciardi, Status of $\left|V_{c b}\right|$ and $\left|V_{u b}\right| C K M$ matrix elements, arXiv: 1412.4288.

[16] G. Ricciardi, Determination of the CKM matrix elements $|V(x b)|$, Mod.Phys.Lett. A28 (2013) 1330016, [arXiv:1305.2844].

[17] A. Alberti, P. Gambino, K. J. Healey, and S. Nandi, Precision determination of the CKM element $V_{c b}, \operatorname{arXiv}: 1411.6560$.

[18] S. Aoki, Y. Aoki, C. Bernard, T. Blum, G. Colangelo, et al., Review of lattice results concerning low-energy particle physics, Eur.Phys.J. C74 (2014), no. 9 2890, [arXiv:1310.8555].

[19] J. A. Bailey, A. Bazavov, C. Bernard, C. Bouchard, C. DeTar, et al., Update of $\left|V_{c b}\right|$ from the $\bar{B} \rightarrow D^{*} \ell \bar{v}$ form factor at zero recoil with three-flavor lattice $Q C D$, arXiv : 1403.0635.

[20] J. Bailey, A. Bazavov, C. Bernard, C. Bouchard, C. DeTar, et al., $B \rightarrow \pi \ell v$ semileptonic form factors from unquenched lattice QCD and determination of $\left|V_{u b}\right|$, arXiv:1411.6038.

[21] A. Crivellin and S. Pokorski, Can the differences in the determinations of $V_{u b}$ and $V_{c b}$ be explained by New Physics?, Phys.Rev.Lett. 114 (2015), no. 1 011802, [arXiv: 1407.1320$].$

[22] F. U. Bernlochner, Z. Ligeti, and S. Turczyk, New ways to search for right-handed current in $B \rightarrow \rho l \bar{v}$ decay, Phys.Rev. D90 (2014), no. 9 094003, [arXiv: 1408.2516 ]. 
[23] A. J. Buras, D. Buttazzo, J. Girrbach-Noe, and R. Knegjens, $K^{+} \rightarrow \pi^{+} v \bar{v}$ and $K_{L} \rightarrow \pi^{0} v \bar{v}$ in the Standard Model: Status and Perspectives, arXiv: 1503.02693.

[24] CKMfitter Group Collaboration, K. Trabelsi, World average and experimental overview of $\gamma / \varphi_{3}$; presented at CKM 2014, . http://www.ckmfitter.in2p3.fr.

[25] A. Bharucha, D. M. Straub, and R. Zwicky, $B \rightarrow V \ell^{+} \ell^{-}$in the Standard Model from Light-Cone Sum Rules, arXiv:1503.05534.

[26] Heavy Flavor Averaging Group Collaboration, Y. Amhis et al., Averages of B-Hadron, C-Hadron, and tau-lepton properties as of early 2012, arXiv:1207.1158.

http://www.slac.stanford.edu/xorg/hfag.

[27] UTfit Collaboration, M. Bona et al., Model-independent constraints on $\Delta F=2$ operators and the scale of new physics, JHEP 0803 (2008) 049, [arXiv : 0707.0636 ]. Updates available on http: //www. utfit.org.

[28] G. Isidori, Y. Nir, and G. Perez, Flavor Physics Constraints for Physics Beyond the Standard Model, Ann.Rev.Nucl.Part.Sci. 60 (2010) 355, [arXiv: 1002 . 0900 ].

[29] J. Charles, S. Descotes-Genon, Z. Ligeti, S. Monteil, M. Papucci, et al., Future sensitivity to new physics in $B_{d}, B_{s}$ and K mixings, Phys.Rev. D89 (2014) 033016, [arXiv: 1309.2293 ].

[30] W. Buchmuller and D. Wyler, Effective Lagrangian Analysis of New Interactions and Flavor Conservation, Nucl.Phys. B268 (1986) 621-653.

[31] B. Grzadkowski, M. Iskrzynski, M. Misiak, and J. Rosiek, Dimension-Six Terms in the Standard Model Lagrangian, JHEP 1010 (2010) 085, [arXiv: 1008 . 4884].

[32] R. Alonso, B. Grinstein, and J. Martin Camalich, $S U(2) \times U(1)$ gauge invariance and the shape of new physics in rare B decays, Phys.Rev.Lett. 113 (2014) 241802, [arXiv: 1407.7044 ].

[33] G. Hiller and M. Schmaltz, $R_{K}$ and future $b \rightarrow$ sll BSM opportunities, Phys.Rev. D90 (2014) 054014, [arXiv:1408.1627].

[34] A. J. Buras, J. Girrbach-Noe, C. Niehoff, and D. M. Straub, $B \rightarrow K^{(*)} v \bar{v}$ decays in the Standard Model and beyond, JHEP 1502 (2015) 184, [arXiv: 1409.4557 ].

[35] A. J. Buras, Minimal flavour violation and beyond: Towards a flavour code for short distance dynamics, Acta Phys.Polon. B41 (2010) 2487-2561, [arXiv:1012.1447].

[36] A. J. Buras and J. Girrbach, BSM models facing the recent LHCb data: A First look, Acta Phys.Polon. B43 (2012) 1427, [arXiv:1204.5064].

[37] A. J. Buras, Relations between $\Delta M_{s, d}$ and $B_{s, d} \rightarrow \mu^{+} \mu^{-}$in models with minimal flavour violation, Phys. Lett. B566 (2003) 115-119, [hep-ph/ 0303060$].$

[38] P. Colangelo, F. De Fazio, P. Santorelli, and E. Scrimieri, Rare $B \rightarrow K^{(*)} v \bar{v}$ decays at B factories, Phys.Lett. B395 (1997) 339-344, [hep-ph/9610297].

[39] G. Buchalla, G. Hiller, and G. Isidori, Phenomenology of non-standard Z couplings in exclusive semileptonic $b \rightarrow$ s transitions, Phys. Rev. D63 (2001) 014015, [hep-ph/ 0006136 ].

[40] W. Altmannshofer, A. J. Buras, D. M. Straub, and M. Wick, New strategies for New Physics search in $B \rightarrow K^{*} v \bar{v}, B \rightarrow K v \bar{v}$ and $B \rightarrow X_{s} v \bar{v}$ decays, JHEP 04 (2009) 022, [arXiv: 0902.0160 ].

[41] A. J. Buras, F. De Fazio, and J. Girrbach, The Anatomy of Z' and Z with Flavour Changing Neutral Currents in the Flavour Precision Era, JHEP 1302 (2013) 116, [arXiv:1211.1896]. 
[42] P. Biancofiore, P. Colangelo, F. De Fazio, and E. Scrimieri, Exclusive $b \rightarrow s v \bar{v}$ induced transitions in $R S_{c}$ model, arXiv:1408.5614.

[43] G. Buchalla and A. J. Buras, $\sin 2 \beta$ from $k \rightarrow \pi v \bar{v}$, Phys. Lett. B333 (1994) 221-227, [hep-ph/9405259].

[44] A. J. Buras and R. Fleischer, Bounds on the unitarity triangle, $\sin 2 \beta$ and $K \rightarrow \pi v \bar{v}$ decays in models with minimal flavor violation, Phys. Rev. D64 (2001) 115010, [hep-ph/ 0104238 ].

[45] T. Blum, P. Boyle, N. Christ, J. Frison, N. Garron, et al., $K \rightarrow \pi \pi \Delta I=3 / 2$ decay amplitude in the continuum limit, arXiv:1502.00263.

[46] A. J. Buras, J.-M. Gérard, and W. A. Bardeen, Large N Approach to Kaon Decays and Mixing 28 Years Later: $\Delta I=1 / 2$ Rule, $\hat{B}_{K}$ and $\Delta M_{K}$, Eur.Phys.J. C74 (2014), no. 5 2871, [arXiv: 1401.1385$]$.

[47] U. Nierste, S. Trine, and S. Westhoff, Charged-Higgs effects in a new $B \rightarrow D \tau v$ differential decay distribution, Phys.Rev. D78 (2008) 015006, [arXiv : 0801.4938 ].

[48] J. F. Kamenik and F. Mescia, $B \rightarrow D \tau v$ Branching Ratios: Opportunity for Lattice QCD and Hadron Colliders, Phys.Rev. D78 (2008) 014003, [arXiv: 0802.3790$].$

[49] BaBar Collaboration, J. Lees et al., Evidence for an excess of $\bar{B} \rightarrow D^{(*)} \tau^{-} \bar{v}_{\tau}$ decays, Phys.Rev.Lett. 109 (2012) 101802, [arXiv:1205.5442].

[50] S. Fajfer, J. F. Kamenik, and I. Nisandzic, On the $B \rightarrow D^{*} \tau \bar{v}_{\tau}$ Sensitivity to New Physics, Phys.Rev. D85 (2012) 094025, [arXiv: 1203.2654 ].

[51] S. Fajfer, J. F. Kamenik, I. Nisandzic, and J. Zupan, Implications of Lepton Flavor Universality Violations in B Decays, Phys.Rev.Lett. 109 (2012) 161801, [arXiv:1206.1872].

[52] A. Crivellin, C. Greub, and A. Kokulu, Explaining $B \rightarrow D \tau \nu, B \rightarrow D^{*} \tau \nu$ and $B \rightarrow \tau \nu$ in a $2 H D M$ of type III, Phys.Rev. D86 (2012) 054014, [arXiv:1206.2634].

[53] A. Crivellin, A. Kokulu, and C. Greub, Flavor-phenomenology of two-Higgs-doublet models with generic Yukawa structure, Phys.Rev. D87 (2013) 094031, [arXiv:1303.5877].

[54] P. Ko, Y. Omura, and C. Yu, $B \rightarrow D^{(*)} \tau \nu$ and $B \rightarrow \tau v$ in chiral U(1)' models with flavored multi Higgs doublets, JHEP 1303 (2013) 151, [arXiv:1212.4607].

[55] A. Crivellin, C. Greub, and A. Kokulu, Flavour-violation in two-Higgs-doublet models, PoS EPS-HEP2013 (2013) 338, [arXiv:1309.4806].

[56] S. Fajfer and I. Nisandzic, Theory of $B \rightarrow \tau \nu$ and $B \rightarrow D^{*} \tau \nu$, arXiv:1301.1167.

[57] MEG Collaboration, J. Adam et al., New constraint on the existence of the $\mu^{+} \rightarrow e^{+} \gamma$ decay, Phys.Rev.Lett. 110 (2013) 201801, [arXiv:1303.0754].

[58] R. Barlow, The PRISM/PRIME project, Nucl.Phys.Proc.Suppl. 218 (2011) 44-49.

[59] M. Raidal et al., Flavour physics of leptons and dipole moments, Eur. Phys. J. C57 (2008) 13-182, [arXiv:0801.1826].

[60] T. Feldmann, Lepton Flavour Violation Theory, PoS BEAUTY2011 (2011) 017, [arXiv:1105.2139].

[61] A. Ibarra, Neutrino physics and lepton flavour violation: A theoretical overview, Nuovo Cim. C033N5 (2010) 67-75. 
[62] R. H. Bernstein and P. S. Cooper, Charged Lepton Flavor Violation: An Experimenter's Guide, Phys.Rept. 532 (2013) 27-64, [arXiv:1307.5787].

[63] M. Blanke, A. J. Buras, B. Duling, A. Poschenrieder, and C. Tarantino, Charged Lepton Flavour Violation and $(g-2)_{\mu}$ in the Littlest Higgs Model with T-Parity: a clear Distinction from Supersymmetry, JHEP 05 (2007) 013, [hep-ph/ 0702136$].$

[64] J. Engel, M. J. Ramsey-Musolf, and U. van Kolck, Electric Dipole Moments of Nucleons, Nuclei, and Atoms: The Standard Model and Beyond, Prog.Part.Nucl.Phys. 71 (2013) 21-74, [arXiv:1303.2371].

[65] M. Pospelov and A. Ritz, Electric dipole moments as probes of new physics, Annals Phys. 318 (2005) 119-169, [hep-ph/ 0504231$].$

[66] B. Batell, Flavor-diagonal CP violation, Eur.Phys.J. C72 (2012) 2127.

[67] M. Blanke, Flavour Physics Beyond the Standard Model: Recent Developments and Future Perspectives, arXiv:1412.1003.

[68] M. Blanke and A. J. Buras, Lower bounds on $\Delta M_{s, d}$ from constrained minimal flavour violation, JHEP 0705 (2007) 061, [hep-ph / 0610037 ].

[69] A. J. Buras and R. Buras, A Lower bound on sin 2 beta from minimal flavor violation, Phys.Lett. B501 (2001) 223-230, [hep-ph/ 0008273$]$.

[70] A. J. Buras, P. Gambino, M. Gorbahn, S. Jager, and L. Silvestrini, Universal unitarity triangle and physics beyond the standard model, Phys. Lett. B500 (2001) 161-167, [hep-ph/ 0007085 ].

[71] A. J. Buras, Minimal flavor violation, Acta Phys. Polon. B34 (2003) 5615-5668, [hep-ph/0310208].

[72] T. Hurth, G. Isidori, J. F. Kamenik, and F. Mescia, Constraints on New Physics in MFV models: A Model-independent analysis of $\Delta F=1$ processes, Nucl. Phys. $\mathbf{B 8 0 8}$ (2009) 326-346, [arXiv:0807.5039].

[73] R. S. Chivukula and H. Georgi, Composite technicolor standard model, Phys. Lett. B188 (1987) 99.

[74] L. J. Hall and L. Randall, Weak scale effective supersymmetry, Phys. Rev. Lett. 65 (1990) 2939-2942.

[75] G. D’Ambrosio, G. F. Giudice, G. Isidori, and A. Strumia, Minimal flavour violation: An effective field theory approach, Nucl. Phys. B645 (2002) 155-187, [hep-ph/0207036].

[76] ETM Collaboration, N. Carrasco et al., B-physics from $N_{f}=2$ tmQCD: the Standard Model and beyond, JHEP 1403 (2014) 016, [arXiv: 1308 .1851].

[77] T. Hermann, M. Misiak, and M. Steinhauser, Three-loop QCD corrections to $B_{S} \rightarrow \mu^{+} \mu^{-}$, JHEP 1312 (2013) 097, [arXiv:1311.1347].

[78] C. Bobeth, M. Gorbahn, and E. Stamou, Electroweak Corrections to $B_{s, d} \rightarrow \ell^{+} \ell^{-}$, Phys.Rev. D89 (2014) 034023, [arXiv:1311.1348].

[79] C. Bobeth, M. Gorbahn, T. Hermann, M. Misiak, E. Stamou, et al., $B_{s, d} \rightarrow \ell^{+} \ell^{-}$in the Standard Model with Reduced Theoretical Uncertainty, Phys.Rev.Lett. 112 (2014) 101801, [arXiv:1311.0903].

[80] CMS Collaboration, LHCb Collaboration Collaboration, V. Khachatryan et al., Observation of the rare $B_{s}^{0} \rightarrow \mu^{+} \mu^{-}$decay from the combined analysis of CMS and LHCb data, arXiv: 1411.4413. 
[81] S. Descotes-Genon, J. Matias, and J. Virto, An analysis of $B_{d, s}$ mixing angles in presence of New Physics and an update of $B_{S} \rightarrow K^{0 *} \bar{K}^{0 *}$, Phys.Rev. D85 (2012) 034010, [arXiv:1111.4882].

[82] K. De Bruyn, R. Fleischer, R. Knegjens, P. Koppenburg, M. Merk, et al., Branching Ratio Measurements of $B_{s}$ Decays, Phys.Rev. D86 (2012) 014027, [arXiv: 1204.1735 ].

[83] K. De Bruyn, R. Fleischer, R. Knegjens, P. Koppenburg, M. Merk, et al., Probing New Physics via the $B_{s}^{0} \rightarrow \mu^{+} \mu^{-}$Effective Lifetime, Phys.Rev.Lett. 109 (2012) 041801, [arXiv: 1204.1737 ].

[84] LHCb Collaboration Collaboration, R. Aaij et al., Precision measurement of CP violation in $B_{s}^{0} \rightarrow J / \psi K^{+} K^{-}$decays, arXiv:1411.3104.

[85] A. J. Buras and J. Girrbach, Stringent Tests of Constrained Minimal Flavour Violation through $\Delta F=2$ Transitions, The European Physical Journal C 9 (73) 2013, [arXiv:1304.6835].

[86] A. Bevan, M. Bona, M. Ciuchini, D. Derkach, E. Franco, et al., Standard Model updates and new physics analysis with the Unitarity Triangle fit, arXiv:1411.7233.

[87] A. J. Buras and J. Girrbach, On the Correlations between Flavour Observables in Minimal U(2) ${ }^{3}$ Models, JHEP 1301 (2013) 007, [arXiv: 1206 . 3878].

[88] R. Barbieri, P. Campli, G. Isidori, F. Sala, and D. M. Straub, B-decay CP-asymmetries in SUSY with a U(2) ${ }^{3}$ flavour symmetry, Eur.Phys.J. C71 (2011) 1812, [arXiv:1108.5125].

[89] R. Barbieri, D. Buttazzo, F. Sala, and D. M. Straub, Flavour physics from an approximate $U(2)^{3}$ symmetry, JHEP 1207 (2012) 181, [arXiv: 1203.4218 ].

[90] A. Crivellin, L. Hofer, and U. Nierste, The MSSM with a Softly Broken U (2) ${ }^{3}$ Flavor Symmetry, PoS EPS-HEP2011 (2011) 145, [arXiv:1111.0246].

[91] R. Barbieri, D. Buttazzo, F. Sala, and D. M. Straub, Flavour physics and flavour symmetries after the first LHC phase, JHEP 1405 (2014) 105, [arXiv: 1402 . 6677].

[92] A. J. Buras, J. Girrbach, and R. Ziegler, Particle-Antiparticle Mixing, CP Violation and Rare K and B Decays in a Minimal Theory of Fermion Masses, JHEP 1304 (2013) 168, [arXiv: 1301.5498 ].

[93] A. J. Buras and J. Girrbach, Left-handed Z' and Z FCNC quark couplings facing new $b \rightarrow s \mu^{+} \mu^{-}$ data, JHEP 1312 (2013) 009, [arXiv:1309.2466].

[94] A. J. Buras, R. Fleischer, J. Girrbach, and R. Knegjens, Probing New Physics with the $B_{s} \rightarrow \mu^{+} \mu^{-}$ Time-Dependent Rate, JHEP 1307 (2013) 77, [arXiv: 1303.3820 ].

[95] A. J. Buras, F. De Fazio, J. Girrbach, R. Knegjens, and M. Nagai, The Anatomy of Neutral Scalars with FCNCs in the Flavour Precision Era, JHEP 1306 (2013) 111, [arXiv: 1303.3723 ].

[96] A. J. Buras, F. De Fazio, J. Girrbach, and M. V. Carlucci, The Anatomy of Quark Flavour Observables in 331 Models in the Flavour Precision Era, JHEP 1302 (2013) 023, [arXiv:1211.1237].

[97] A. J. Buras, F. De Fazio, and J. Girrbach, 331 models facing new $b \rightarrow s \mu^{+} \mu^{-}$data, JHEP 1402 (2014) 112, [arXiv:1311.6729].

[98] A. J. Buras, F. De Fazio, and J. Girrbach-Noe, Z-Z' mixing and Z-mediated FCNCs in $S U(3)_{C} \times S U(3)_{L} \times U(1)_{X}$ Models, JHEP 1408 (2014) 039, [arXiv: 1405.3850 ].

[99] S. Descotes-Genon, L. Hofer, J. Matias, and J. Virto, On the impact of power corrections in the prediction of $B \rightarrow K^{*} \mu^{+} \mu^{-}$observables, JHEP 1412 (2014) 125, [arXiv: 1407.8526 ]. 
[100] W. Altmannshofer and D. M. Straub, State of new physics in $b \rightarrow$ s transitions, arXiv:1411.3161.

[101] S. L. Glashow, D. Guadagnoli, and K. Lane, Lepton Flavor Violation in B Decays?, arXiv:1411.0565.

[102] W. Altmannshofer and D. M. Straub, Implications of $b \rightarrow$ s measurements, arXiv:1503.06199.

[103] S. Descotes-Genon, J. Matias, and J. Virto, Understanding the $B \rightarrow K^{*} \mu^{+} \mu^{-}$Anomaly, Phys. Rev. D 88, 074002 (2013) [arXiv: 1307.5683$].$

[104] W. Altmannshofer and D. M. Straub, New physics in $B \rightarrow K^{*} \mu \mu$ ?, arXiv:1308.1501.

[105] F. Beaujean, C. Bobeth, D. van Dyk, and C. Wacker, Bayesian Fit of Exclusive $b \rightarrow$ sēl Decays: The Standard Model Operator Basis, JHEP 1208 (2012) 030, [arXiv: 1205.1838 ].

[106] C. Bobeth, G. Hiller, and D. van Dyk, General Analysis of $\bar{B} \rightarrow \bar{K}^{(*)} \ell^{+} \ell^{-}$Decays at Low Recoil, Phys.Rev. D87 (2013) 034016, [arXiv:1212.2321].

[107] F. Beaujean, C. Bobeth, and D. van Dyk, Comprehensive Bayesian analysis of rare (semi)leptonic and radiative B decays, Eur.Phys.J. C74 (2014), no. 6 2897, [arXiv:1310 .2478].

[108] A. Datta, M. Duraisamy, and D. Ghosh, Explaining the $B \rightarrow K^{*} \mu^{+} \mu^{-}$data with scalar interactions, Phys.Rev. D89 (2014), no. 7 071501, [arXiv:1310.1937].

[109] D. Ghosh, M. Nardecchia, and S. Renner, Hint of Lepton Flavour Non-Universality in B Meson Decays, arXiv:1408.4097.

[110] T. Hurth, F. Mahmoudi, and S. Neshatpour, Global fits to $b \rightarrow$ sll data and signs for lepton non-universality, JHEP 1412 (2014) 053, [arXiv: 1410.4545$].$

[111] A. Khodjamirian, T. Mannel, A. Pivovarov, and Y.-M. Wang, Charm-loop effect in $B \rightarrow K^{(*)} \ell^{+} \ell^{-}$ and $B \rightarrow K^{*} \gamma$, JHEP 1009 (2010) 089, [arXiv: 1006.4945$]$.

[112] M. Beylich, G. Buchalla, and T. Feldmann, Theory of $B \rightarrow K^{(*)} l^{+} l^{-}$decays at high $q^{2}:$ OPE and quark-hadron duality, Eur.Phys.J. C71 (2011) 1635, [arXiv:1101.5118].

[113] J. Matias, On the S-wave pollution of $B \rightarrow K^{*} l^{+} l^{-}$observables, Phys.Rev. D86 (2012) 094024, [arXiv:1209.1525].

[114] S. Jager and J. M. Camalich, On B $\rightarrow V \ell \ell$ at small dilepton invariant mass, power corrections, and new physics, JHEP 1305 (2013) 043, [arXiv: 1212 . 2263].

[115] C. Hambrock, G. Hiller, S. Schacht, and R. Zwicky, $B \rightarrow K^{*}$ Form Factors from Flavor Data to QCD and Back, Phys.Rev. D89 (2014) 074014, [arXiv: 1308 .4379].

[116] R. R. Horgan, Z. Liu, S. Meinel, and M. Wingate, Calculation of $B^{0} \rightarrow K^{* 0} \mu^{+} \mu^{-}$and $B_{s}^{0} \rightarrow \phi \mu^{+} \mu^{-}$ observables using form factors from lattice QCD, Phys.Rev.Lett. 112 (2014) 212003, [arXiv:1310.3887].

[117] R. R. Horgan, Z. Liu, S. Meinel, and M. Wingate, Lattice QCD calculation of form factors describing the rare decays $B \rightarrow K^{*} \ell^{+} \ell^{-}$and $B_{s} \rightarrow \phi \ell^{+} \ell^{-}$, Phys.Rev. D89 (2014) 094501, [arXiv:1310.3722].

[118] S. Jager and J. M. Camalich, Reassessing the discovery potential of the $B \rightarrow K^{*} \ell^{+} \ell^{-}$decays in the large-recoil region: SM challenges and BSM opportunities, arXiv:1412.3183.

[119] W. Altmannshofer, S. Gori, M. Pospelov, and I. Yavin, Dressing $L_{\mu}-L_{\tau}$ in Color, arXiv:1403.1269. 
[120] B. Bhattacharya, A. Datta, D. London, and S. Shivashankara, Simultaneous Explanation of the $R_{K}$ and $R\left(D^{(*)}\right)$ Puzzles, Phys.Lett. B742 (2015) 370-374, [arXiv: 1412.7164 ].

[121] A. Crivellin, G. D’Ambrosio, and J. Heeck, Explaining $h \rightarrow \mu^{ \pm} \tau^{\mp}, B \rightarrow K^{*} \mu^{+} \mu^{-}$and $B \rightarrow K \mu^{+} \mu^{-} / B \rightarrow K e^{+} e^{-}$in a two-Higgs-doublet model with gauged $L_{\mu}-L_{\tau}$, arXiv:1501.00993.

[122] A. Crivellin, G. D’Ambrosio, and J. Heeck, Addressing the LHC flavour anomalies with horizontal gauge symmetries, arXiv:1503.03477.

[123] R. Gauld, F. Goertz, and U. Haisch, On minimal $Z^{\prime}$ explanations of the $B \rightarrow K^{*} \mu^{+} \mu^{-}$anomaly, arXiv:1308.1959.

[124] R. Gauld, F. Goertz, and U. Haisch, An explicit Z'-boson explanation of the $B \rightarrow K^{*} \mu^{+} \mu^{-}$anomaly, JHEP 1401 (2014) 069, [arXiv: 1310 .1082].

[125] S. Biswas, D. Chowdhury, S. Han, and S. J. Lee, Explaining the lepton non-universality at the LHCb and CMS within a unified framework, JHEP 1502 (2015) 142, [arXiv: 1409.0882 ].

[126] B. Gripaios, M. Nardecchia, and S. Renner, Composite leptoquarks and anomalies in B-meson decays, arXiv:1412.1791.

[127] S. Sahoo and R. Mohanta, Scalar leptoquarks and the rare B meson decays, arXiv:1501.05193.

[128] I. de Medeiros Varzielas and G. Hiller, Clues for flavor from rare lepton and quark decays, arXiv:1503.01084.

[129] F. Pisano and V. Pleitez, An SU(3) x U(1) model for electroweak interactions, Phys.Rev. D46 (1992) 410-417, [hep-ph/9206242].

[130] P. H. Frampton, Chiral dilepton model and the flavor question, Phys. Rev. Lett. 69 (1992) 2889-2891.

[131] R. Martinez and F. Ochoa, Constraints on 3-3-1 models with electroweak Z pole observables and $Z^{\prime}$ search at the LHC, Phys.Rev. D90 (2014), no. 1 015028, [arXiv: 1405.4566 ].

[132] J. F. Kamenik and C. Smith, Tree-level contributions to the rare decays $B^{+} \rightarrow \pi^{+} v \bar{v}, B^{+} \rightarrow K^{+} v \bar{v}$, and $B^{+} \rightarrow K^{*+} v \bar{v}$ in the Standard Model, Phys.Lett. B680 (2009) 471-475, [arXiv: 0908.1174$]$.

[133] G. A. Rinella, R. Aliberti, F. Ambrosino, B. Angelucci, A. Antonelli, et al., Prospects for $K^{+} \rightarrow \pi^{+} v \bar{v}$ at CERN in NA62, arXiv:1411.0109.

[134] A. Romano, The $K^{+} \rightarrow \pi^{+} v \bar{v}$ decay in the NA62 experiment at CERN, arXiv:1411.6546.

[135] T. Komatsubara, Experiments with K-Meson Decays, Prog.Part.Nucl.Phys. 67 (2012) 995-1018, [arXiv:1203.6437].

[136] for the KOTO collaboration Collaboration, K. Shiomi, $K_{L}^{0} \rightarrow \pi^{0} v \bar{v}$ at KOTO, arXiv:1411.4250.

[137] G. Buchalla and A. J. Buras, Qcd corrections to rare $k$ and $b$ decays for arbitrary top quark mass, Nucl. Phys. B400 (1993) 225-239.

[138] M. Misiak and J. Urban, QCD corrections to FCNC decays mediated by $Z$ penguins and $W$ boxes, Phys.Lett. B451 (1999) 161-169, [hep-ph/9901278].

[139] G. Buchalla and A. J. Buras, The rare decays $K \rightarrow \pi v \bar{v}, B \rightarrow X v \bar{v}$ and $B \rightarrow \ell^{+} \ell^{-}:$An Update, Nucl.Phys. B548 (1999) 309-327, [hep-ph/9901288]. 
[140] A. J. Buras, M. Gorbahn, U. Haisch, and U. Nierste, The rare decay $K^{+} \rightarrow \pi^{+} v \bar{v}$ at the next-to-next-to-leading order in QCD, Phys. Rev. Lett. 95 (2005) 261805, [hep-ph/ 0508165 ].

[141] A. J. Buras, M. Gorbahn, U. Haisch, and U. Nierste, Charm quark contribution to $K^{+} \rightarrow \pi^{+} \nu \bar{v}$ at next-to-next-to-leading order, JHEP 11 (2006) 002, [hep-ph / 0603079 ].

[142] M. Gorbahn and U. Haisch, Effective Hamiltonian for non-leptonic $|\Delta F|=1$ decays at NNLO in QCD, Nucl.Phys. B713 (2005) 291-332, [hep-ph / 0411071$].$

[143] J. Brod and M. Gorbahn, Electroweak Corrections to the Charm Quark Contribution to $K^{+} \rightarrow \pi^{+} v \bar{v}$, Phys. Rev. D78 (2008) 034006, [arXiv: 0805.4119$]$.

[144] J. Brod, M. Gorbahn, and E. Stamou, Two-Loop Electroweak Corrections for the $K \rightarrow \pi v \bar{n} u$ Decays, Phys.Rev. D83 (2011) 034030, [arXiv: 1009 .0947].

[145] G. Buchalla and A. J. Buras, Two-loop large- $m_{t}$ electroweak corrections to $k \rightarrow \pi v \bar{v}$ for arbitrary higgs boson mass, Phys. Rev. D57 (1998) 216-223, [hep-ph/9707243].

[146] C. Bouchard, E. Freeland, C. Bernard, C. Chang, A. El-Khadra, et al., Neutral B-meson mixing parameters in and beyond the SM with 2+1 flavor lattice QCD, arXiv:1412.5097.

[147] UTfit Collaboration, M. Bona et al., An Improved Standard Model Prediction Of BR $(B \rightarrow \tau v)$ And Its Implications For New Physics, Phys.Lett. B687 (2010) 61-69, [arXiv: 0908 . 3470 ].

[148] J. Charles, O. Deschamps, S. Descotes-Genon, R. Itoh, H. Lacker, et al., Predictions of selected flavour observables within the Standard Model, Phys.Rev. D84 (2011) 033005, [arXiv:1106.4041].

[149] A. J. Buras, F. De Fazio, and J. Girrbach, $\Delta I=1 / 2$ rule, $\varepsilon^{\prime} / \varepsilon$ and $K \rightarrow \pi v \bar{v}$ in $Z^{\prime}(Z)$ and $G^{\prime}$ models with FCNC quark couplings, Eur.Phys.J. C74 (2014) 2950, [arXiv: 1404.3824 ].

[150] M. Blanke, Insights from the Interplay of $K \rightarrow \pi v \bar{v}$ and $\varepsilon_{K}$ on the New Physics Flavour Structure, Acta Phys.Polon. B41 (2010) 127, [arXiv: 0904 .2528].

[151] A. J. Buras, D. Buttazzo, J. Girrbach-Noe, and R. Knegjens, Can we reach the Zeptouniverse with rare $K$ and $B_{s, d}$ decays?, JHEP 1411 (2014) 121, [arXiv: 1408.0728 ].

[152] Y. Grossman and Y. Nir, $K_{L} \rightarrow \pi^{0} v \bar{v}$ beyond the standard model, Phys. Lett. B398 (1997) 163-168, [hep-ph/9701313].

[153] J. Hewett, H. Weerts, R. Brock, J. Butler, B. Casey, et al., Fundamental Physics at the Intensity Frontier, arXiv:1205.2671.

[154] D. McKeen, M. Pospelov, and A. Ritz, Electric dipole moment signatures of PeV-scale superpartners, Phys.Rev. D87 (2013), no. 11 113002, [arXiv:1303.1172].

[155] T. Moroi and M. Nagai, Probing Supersymmetric Model with Heavy Sfermions Using Leptonic Flavor and CP Violations, Phys.Lett. B723 (2013) 107-112, [arXiv:1303.0668].

[156] T. Moroi, M. Nagai, and T. T. Yanagida, Lepton Flavor Violations in High-Scale SUSY with Right-Handed Neutrinos, Phys.Lett. B728 (2014) 342-346, [arXiv: 1305 . 7357 ].

[157] L. Eliaz, A. Giveon, S. B. Gudnason, and E. Tsuk, Mild-split SUSY with flavor, JHEP 1310 (2013) 136, [arXiv:1306.2956].

[158] A. S. Kronfeld, R. S. Tschirhart, U. Al-Binni, W. Altmannshofer, C. Ankenbrandt, et al., Project X: Physics Opportunities, arXiv:1306.5009. 
[159] A. de Gouvea and P. Vogel, Lepton Flavor and Number Conservation, and Physics Beyond the Standard Model, Prog.Part.Nucl.Phys. 71 (2013) 75-92, [arXiv:1303.4097].

[160] W. Altmannshofer, R. Harnik, and J. Zupan, Low Energy Probes of PeV Scale Sfermions, JHEP 1311 (2013) 202, [arXiv:1308.3653]. 